\title{
Tensor networks from kinematic space
}

\author{
Bartłomiej Czech, ${ }^{a}$ Lampros Lamprou, ${ }^{a}$ Samuel McCandlish ${ }^{a}$ and James Sully ${ }^{b}$ \\ ${ }^{a}$ Stanford Institute for Theoretical Physics, Department of Physics, \\ Stanford University, Stanford, CA 94305, U.S.A. \\ ${ }^{b}$ SLAC, Stanford University, \\ Menlo Park, CA 94025, U.S.A. \\ E-mail: czech@stanford.edu, llamprou@stanford.edu, \\ samsamoa@stanford.edu, jsully@slac.stanford.edu
}

ABSTRACT: We point out that the MERA network for the ground state of a 1+1-dimensional conformal field theory has the same structural features as kinematic space - the geometry of CFT intervals. In holographic theories kinematic space becomes identified with the space of bulk geodesics studied in integral geometry. We argue that in these settings MERA is best viewed as a discretization of the space of bulk geodesics rather than of the bulk geometry itself. As a test of this kinematic proposal, we compare the MERA representation of the thermofield-double state with the space of geodesics in the two-sided BTZ geometry, obtaining a detailed agreement which includes the entwinement sector. We discuss how the kinematic proposal can be extended to excited states by generalizing MERA to a broader class of compression networks.

KEYwords: AdS-CFT Correspondence, Gauge-gravity correspondence

ARXIV EPRINT: 1512.01548 


\section{Contents}

1 Introduction $\quad 2$

2 Review 4

2.1 Kinematic space 4

2.1.1 Crofton's formula in flat space 4

2.1.2 Crofton's formula in holographic geometries 4

2.1.3 Causal structure and the kinematic metric 6

2.1.4 Conditional mutual information in kinematic space $\quad 7$

2.2 The MERA network $\quad 7$

2.2.1 Tensor network generalities $\quad 8$

2.2.2 Structure of the MERA network 8

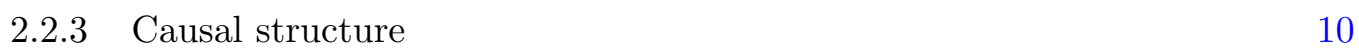

$\begin{array}{ll}\text { 2.2.4 Entanglement entropies from cut-counting } & 12\end{array}$

3 MERA and kinematic geometry 13

$\begin{array}{lll}3.1 & \text { Partial order of MERA and kinematic causality } & 13\end{array}$

$\begin{array}{lll}3.1 .1 & \text { Spacelike versus timelike paths } & 14\end{array}$

$\begin{array}{lll}3.1 .2 & \text { Representation of symmetries } & 15\end{array}$

$\begin{array}{lll}3.2 & \text { Localization of information } & 16\end{array}$

$\begin{array}{lll}\text { 3.2.1 Crofton form and volumes in MERA } & 16\end{array}$

$\begin{array}{ll}3.2 .2 \text { Entanglement entropy } & 18\end{array}$

$\begin{array}{lll}3.3 & \text { MERA as renormalization } & 20\end{array}$

$\begin{array}{lll}\text { 3.3.1 Coarse-graining with MERA } & 20\end{array}$

$\begin{array}{lll}\text { 3.3.2 Real space RG and causal cuts } & 21\end{array}$

4 MERA for boundary gravitons and two-sided black holes 22

$\begin{array}{ll}4.1 \text { Boundary gravitons } & 22\end{array}$

4.2 The thermofield double state and the two-sided BTZ black hole 23

4.3 The quotient MERA is the kinematic space of the two-sided black hole 24

5 Toward excited states: geometry as compression 28

$\begin{array}{llr}6 & \text { Discussion } & 29\end{array}$ 


\section{Introduction}

Gravitational physics presents us with a paradox. On the one hand, its most successful formulation to date - the general theory of relativity - relies on differential geometry, which emphasizes local dynamics. On the other hand, all gauge-invariant observables in gravity live on the asymptotic boundary and are therefore global in character. While the local approach has been pursued with undiminished success for one century [1], a more global strategy has not yet congealed into a unified formalism. The best developed attempt to fill this gap is the AdS/CFT correspondence [2], which organizes the gauge-invariant quantities in a gravitational spacetime into a field theory living on its asymptotic boundary. A key challenge facing the holographic program - one that has come to the spotlight in recent years [3, 4] - is this: how do we reconcile the CFT-based, global formulation of gravity with the local language of general relativity?

In ref. [5], we outlined a semantically evident answer to this question: to complement Einstein's apparatus of differential geometry, we need an approach based on integral geometry [6]. This beautiful field of mathematics is concerned with translating between local and global properties of geometric spaces. A well-known application is to recover a function from its integrals along straight lines [7], a problem that occurs in seismology [8], medical imaging [9] and the reconstruction of bulk operators in holographic duality [10]. Building up on this technology, we and one collaborator introduced a new entry to the holographic dictionary: operators called OPE blocks, which are dual to integrals of bulk fields along geodesics and homogeneous surfaces [11]. ${ }^{1}$ The present paper, which builds up on [5], focuses on another classic, integral geometric problem: determining the geometry of an asymptotically $\mathrm{AdS}_{3}$ spacetime from data in the dual conformal field theory.

Generally, the input to the reconstruction problem consists of all correlation functions in the CFT. However, recent years have taught us that information-theoretic CFT data are particularly robust probes of the bulk geometry. The foremost among them are entanglement entropies of boundary regions, which compute areas of bulk minimal surfaces [1214]. ${ }^{2}$ In ref. [5], we used entanglement entropies to define an auxiliary, Lorentzian geometry, whose points are in one-to-one correspondence with boundary intervals and, by the RyuTakayanagi proposal [12], with spacelike geodesics in the dual geometry. The resulting object, called kinematic space, is an intermediary in the AdS/CFT translation, providing a natural volume form on the space of bulk geodesics. Integrals of that form compute lengths of all bulk curves in a generalization of the famous Crofton formula, which tells us how likely a dropped needle is to land on a single bathroom tile [26].

But the problems solved by kinematic space are not confined to holographic duality. Even in the absence of a gravitational dual, $d$-dimensional conformal field theory intertwines space and scale (RG direction), as is evident from its global symmetry group $\mathrm{SO}(d, 2)$. One may imagine that kinematic space, which organizes the entanglement structure of a state

\footnotetext{
${ }^{1}$ In the Discussion, we explain how those findings are relevant to the content of the present paper.

${ }^{2}$ Numerous other gravitational quantities also have information-theoretic dual descriptions, including a version of the null energy condition [15], Einstein's equations [16-18], canonical energy [19], lengths of curves [20-22], the triangle inequality [23] and even connectedness of spacetime [24, 25].
} 


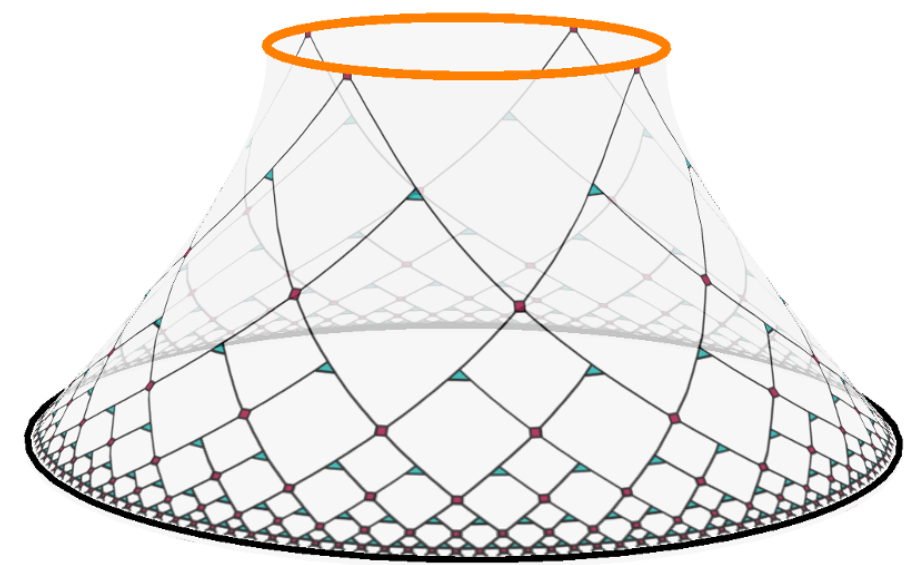

Figure 1. MERA naturally lives on half of two-dimensional de Sitter space, the kinematic space for an equal-time slice of $\mathrm{AdS}_{3}$.

by location and scale, may have already found use in the study of conformal field theories, independently of holographic considerations. If so, in what form has kinematic space previously appeared?

The answer is the Multi-Scale Entanglement Renormalization Ansatz (MERA) [27, 28]. The present paper explains the merits of viewing the MERA network as a discrete version of the vacuum kinematic space. The argument makes crucial use of the auxiliary causal structure of MERA, which originates from working with unitary and isometric tensors as part of the ansatz. This causal structure was independently exploited to argue that MERA most naturally lives on de Sitter space [29]. Our key insight is to recognize that this de Sitter space is the vacuum kinematic space, which carries an information metric determined by entanglement. This allows us to propose a generalization of MERA to excited states [30]. We stress that our results do not preclude other types of networks, for example ones studied in [31-34], from discretizing a time slice of the dual geometry directly.

The connection between holographic duality and tensor networks has been an object of intense interest; see e.g. [31-42]. The initial impetus was due to Swingle who observed that the MERA network resembles in many ways an equal time slice of anti-de Sitter space $[35,36]$. We will explain that relating MERA to kinematic space enjoys the same benefits as Swingle's original proposal, but it adds to them a sizable list of its own. At the same time, we emphasize that the relation between MERA and holographic duality is primarily qualitative; its main purpose is to stimulate progress by offering a compelling analogy. This modus operandi has been extremely fruitful thus far, but we believe that further MERA-inspired progress hinges on other aspects of Vidal's ansatz, which are only clarified with reference to kinematic space.

Reading guide. In an effort to keep the paper self-contained, we begin with a review of integral geometry and MERA (section 2). Sections 3 and 4 contain the main arguments for identifying MERA with the kinematic geometry. Section 3 is set in the broad context of the ground state MERA while section 4 discusses the recently reported [43] MERA 
construction of the thermofield double state. Section 5 briefly motivates the results of the second part of this work, which will be presented in [30]. Section 6 summarizes our core arguments and discusses the main results of this work.

\section{Review}

We begin by reviewing the properties of kinematic space and the MERA tensor network. The reader is encouraged to look for commonalities.

\subsection{Kinematic space}

A more complete discussion of the ensuing material was given in [5].

\subsubsection{Crofton's formula in flat space}

Crofton's formula states that the length of a curve is measured by the number of straight lines that intersect it. To state this result formally, we need to clarify how to count straight lines. Straight lines on the plane form a two-dimensional manifold $K$ known as 'kinematic space.' To quantify 'how many' straight lines $g$ satisfy some condition, we need a homogeneous measure $\mathcal{D} g$ on kinematic space. Using translations and rotations fixes the measure, up to a multiplicative constant, to be

$$
\mathcal{D} g=d p \wedge d \theta
$$

Here $p$ is the distance of the straight line from the origin and $\theta$ is the angle it makes with some fixed axis. Allowing $p$ to be negative extends the measure to the set of oriented straight lines.

Crofton's formula states that, for every curve $\gamma$ of finite length,

$$
\text { length of } \gamma=\frac{1}{4} \int_{K} n(g, \gamma) \mathcal{D} g \text {. }
$$

Here $n(g, \gamma)$ is the number of intersections between the straight line $g$ and $\gamma$. This result can be used to solve Buffon's needle problem [26], which we referenced in the Introduction. Our primary interest is in an extension of this formula to holographic spacetimes.

\subsubsection{Crofton's formula in holographic geometries}

To extend eq. (2.2) to a static slice of a holographic geometry, one need only supply the correct measure on the generalized kinematic space. In pure $\mathrm{AdS}_{3}$ of curvature radius $L$, the measure is again uniquely selected by invariance under the isometries of $\mathbb{H}_{2}$ [44], and takes the form

$$
\mathcal{D} g=\frac{L d \theta \wedge d \alpha}{\sin ^{2} \alpha}
$$

This expression gives a 'density of geodesics' near a geodesic centered at a boundary point $\theta$, with opening angle $\alpha$ (see figure 2). Looking ahead to a connection with the MERA network, we note that $\theta$ and $\alpha$ play the role of position and scale in the CFT, respectively. 

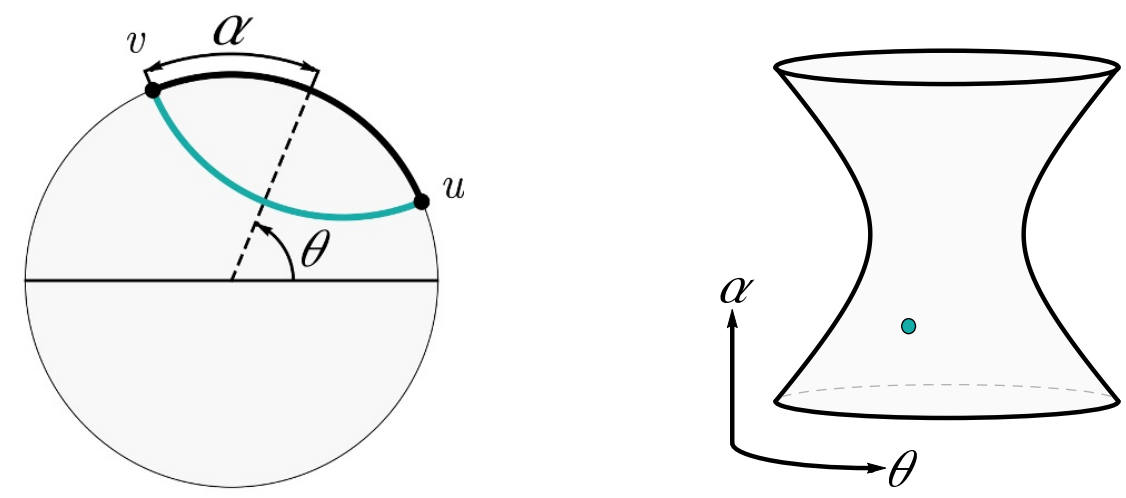

Figure 2. The kinematic coordinates $\alpha$ and $\theta$ correspond to the half-opening angle of the geodesic and the angular location of its center-point repectively. Geodesics in the hyperbolic plane are mapped to points on kinematic space.

In a general static geometry, the measure cannot be found from symmetry alone. In ref. [5], we showed that when the tangent bundle of a bulk time-slice is covered by boundary-anchored geodesics, the measure takes a remarkably simple form,

$$
\mathcal{D} g=\frac{\partial^{2} S(u, v)}{\partial u \partial v} d u \wedge d v,
$$

where $S(u, v)$ is the length of a geodesic connecting the boundary points $u$ and $v$. Here, we have defined 'light-cone' coordinates on kinematic space,

$$
u=\theta-\alpha \quad \text { and } \quad v=\theta+\alpha,
$$

which label a geodesic by its asymptotic endpoints. Then eq. (2.4) agrees with eq. (2.3) after substituting $S(u, v)=2 L \log \sin \frac{v-u}{2}+$ const.

When $S(u, v)$ refers to the length of the shortest geodesic connecting points $u$ and $v$ satisfying the homology condition, its length in units of $4 G$ is the entanglement entropy of the boundary interval $(u, v)$ [12]. Thus, it is convenient to divide both sides of eq. (2.2) by $4 G$ and obtain:

$$
\frac{\text { length of } \gamma}{4 G}=\frac{1}{4} \int_{K} n(g, \gamma) \frac{\partial^{2} S_{\text {ent }}(u, v)}{\partial u \partial v} d u \wedge d v
$$

In what follows, we set $4 G \equiv 1$ and do not distinguish between $S(u, v)$ as a length and $S_{\text {ent }}(u, v)$ as an entanglement entropy.

Differential entropy. For a closed curve, it is instructive to carry out the integral in (2.6) explicitly in one direction:

$$
\text { length of } \gamma=-\left.\int_{0}^{2 \pi} d u \frac{\partial S(u, v)}{\partial u}\right|_{v=v(u)}=S_{\text {diff }}
$$

This expression is the differential entropy, first reported in [20] (see also [21]). It localizes on the set of geodesics tangent to $\gamma$, which is the boundary of the set of its intersecting geodesics. The tangency condition appears through $v(u)$, which is defined by demanding that the geodesic connecting $u$ and $v(u)$ be tangent to $\gamma$. 
In section 2.2.4 we will find an analogue of this expression in the cut-counting prescription for estimating entropies in MERA.

\subsubsection{Causal structure and the kinematic metric}

The kinematic space for a static slice of an asymptotically $\mathrm{AdS}_{3}$ geometry has a richer structure than just a density form: it can also be equipped with a metric with mixed signature. To see this, note that the space of geodesics maps naturally to the space of boundary intervals via the $\mathrm{R}-\mathrm{T}$ prescription. The causal structure of kinematic space descends not from the causal structure of $\mathrm{AdS}_{3}$, but from the partial ordering of boundary intervals by containment.

In particular, given two boundary intervals $A, B$ corresponding to two points $a, b$ in kinematic space, we say that $a$ causally precedes $b$ if $A \subset B$. Any pair of geodesics may then be classified as timelike, lightlike, or spacelike-separated:

- Timelike: geodesic $\left(u_{1}, v_{1}\right)$ is said to live in the past of geodesic $\left(u_{2}, v_{2}\right)$ if

$$
\left[u_{1}, v_{1}\right] \subset\left[u_{2}, v_{2}\right]
$$

as intervals on the asymptotic boundary. Note that the direction of kinematic 'time' reverses under changes of orientation. For the same geodesics with opposite orientation, we have $\left[v_{2}, u_{2}\right] \subset\left[v_{1}, u_{1}\right]$.

- Spacelike: geodesics $\left(u_{1}, v_{1}\right)$ and $\left(u_{2}, v_{2}\right)$ are spacelike separated when neither interval contains the other.

- Lightlike: this is the borderline case between spacelike and timelike separation. It occurs when one of the intervals subtended by the geodesics contains the other, but only marginally. This means that the intervals share an endpoint - on the left or on the right:

$$
u_{1}=u_{2} \quad \text { or } \quad v_{1}=v_{2}
$$

This is the reason why above eq. (2.5) we referred to the endpoint coordinates of kinematic space as 'light-cone' coordinates.

Kinematic metric. For a time-slice of pure $\mathrm{AdS}_{3}$, we can now see that symmetry fixes the metric on kinematic space to be the two-dimensional de Sitter metric [23, 44]:

$$
d s_{\text {kin }}^{2}=\frac{L}{\sin ^{2} \alpha}\left(-d \alpha^{2}+d \theta^{2}\right) .
$$

To see this, note that $\mathrm{dS}_{2}$ is the only metric space with $\mathrm{SO}(2,1)$ isometry group that realizes the requisite causal structure. With the coefficient above, the volume form $d^{2} V_{\text {kin }}$ in kinematic space is equal to the geodesic density $\mathcal{D} g$ of eq. (2.3).

Moving to a general asymptotically hyperbolic geometry, specifying the causal structure and the volume form $d^{2} V_{\text {kin }}=\mathcal{D} g$ yields a unique Lorentzian metric:

$$
d s_{\text {kin }}^{2}=\frac{\partial^{2} S(u, v)}{\partial u \partial v} d u d v
$$

The relevance of this metric for reconstructing local features of the bulk geometry was reported in $[5,23]$. 


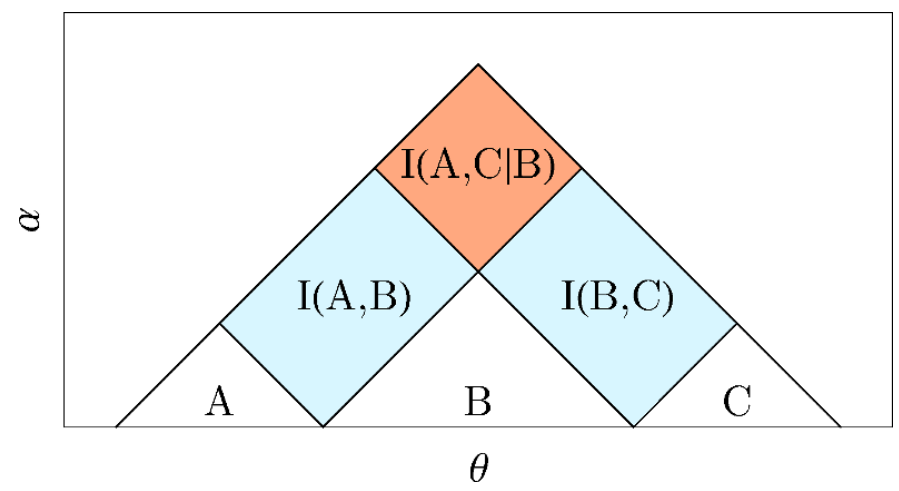

Figure 3. Volumes of causal diamonds in kinematic space compute conditional mutual informations of triples of contiguous intervals. As a special case, causal diamonds with one vertex on the boundary compute mutual informations of adjacent intervals.

\subsubsection{Conditional mutual information in kinematic space}

The volume form (2.4) has a meaning in information theory. Conditional mutual information is defined as the following combination of entanglement entropies:

$$
I(A, C \mid B)=S(A B)+S(B C)-S(A B C)-S(B)
$$

Mutual information $I(A, C)$ is a special case of this quantity, conditioned on $B=\emptyset$. Conditional mutual information is also familiar from the strong subadditivity of entanglement entropy, which guarantees that it is non-negative [45]. For the special choice

$$
A=(u-d u, u) \quad \text { and } \quad B=(u, v) \quad \text { and } \quad C=(v, v+d v),
$$

we have:

$$
S(u-d u, v)+S(u, v+d v)-S(u-d u, v+d v)-S(u, v)=\frac{\partial^{2} S(u, v)}{\partial u \partial v} d u d v=d^{2} V_{\text {kin }} .
$$

Eq. (2.14) states that the Lorentzian area of an infinitesimal causal diamond in kinematic space computes the conditional mutual information of a triple of neighboring intervals (2.13). Owing to the chain rule for conditional mutual information

$$
I(A, C D \mid B)=I(A, C \mid B)+I(A, D \mid B C),
$$

this conclusion automatically extends to all causal diamonds in kinematic space, regardless of size (see figure 3). A special case is a causal diamond with one of its vertices on the boundary of kinematic space, whose volume is equal to the mutual information of the two adjacent intervals. Thus, eq. (2.6) states that the length of any curve on a static slice of an asymptotically $\mathrm{AdS}_{3}$ geometry computes a combination of conditional mutual informations.

\subsection{The MERA network}

Our presentation will be brief, because good reviews exist elsewhere [46, 47]. We highlight those aspects of MERA, which are key for appreciating the connection with kinematic space. A reader familiar with MERA may skip over to section 3. 


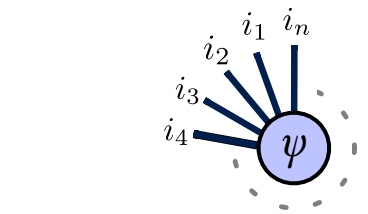

(a)

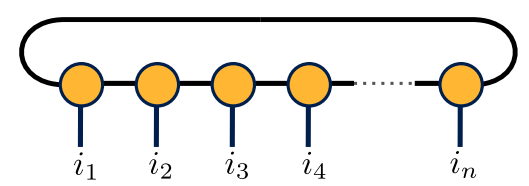

(b)

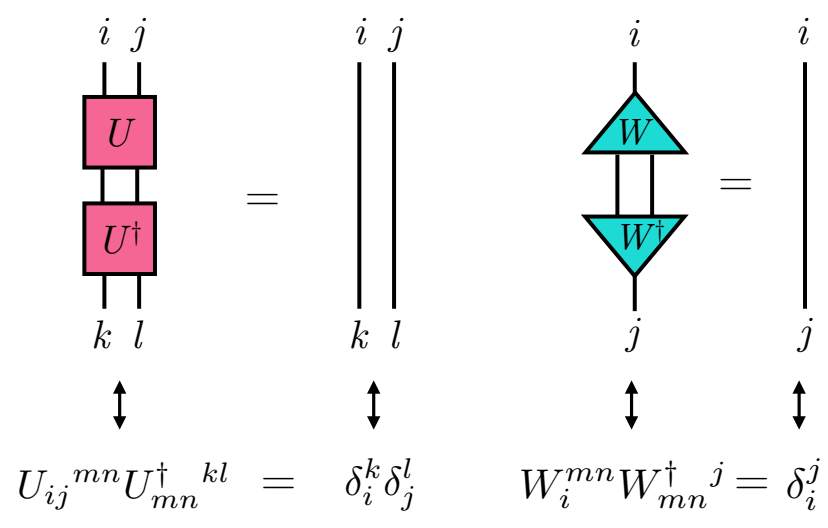

(c)

Figure 4. Examples of tensor networks. (a) A featureless tensor network composed of a single tensor. This can prepare a generic state, as in eq. (2.16). (b) A tensor network composed of a chain of tensors contracted together (a matrix product state). (c) The unitary (resp. isometric) character of the disentanglers and isometries in MERA means that these tensors cancel out when contracted with their hermitian conjugates.

\subsubsection{Tensor network generalities}

The wavefunction of a general $N$-body system defines a tensor with $N$ indices:

$$
|\Psi\rangle=\sum_{i_{1} i_{2} \ldots i_{N}} \Psi_{i_{1} i_{2} \ldots i_{N}}\left|i_{1} i_{2} \ldots i_{N}\right\rangle
$$

The walloping number of components of this tensor -exponential in $N$ - reflects the complexity of an arbitrary many-body wavefunction. However, imposing physical constraints such as locality and symmetry ought to simplify the description of the wavefunction drastically. This simplification is the objective of tensor network techniques.

Tensor networks are graphs, which consist of vertices and edges. Every vertex stands for a tensor with as many indices, as there are edges incident on it. The indices range from 1 to $\chi$, the 'bond dimension' of a given edge. An edge connecting two vertices denotes a common index of two tensors, which is contracted (traced out.) Some examples of tensor networks, including the featureless wavefunction from eq. (2.16), are shown in figure 4 .

\subsubsection{Structure of the MERA network}

The MERA network is a successful ansatz for the ground state wavefunction of a conformal field theory [47]. For a $\mathrm{CFT}_{2}$ - the case of interest in the present paper - it is a twodimensional array of tensors shown in figure 5. While the horizontal direction corresponds to the spatial axis of the CFT, the vertical direction is meant to encode scale (RG direction). In a true CFT, which has no characteristic scale, the vertical direction ought to be infinite. In practice, however, MERA networks are presented with a finite number of layers, which is tantamount to fixing a UV cutoff.

Because the axes of MERA correspond to space and scale, the network provides a graphical representation of renormalization in real space. For example, cutting the network 

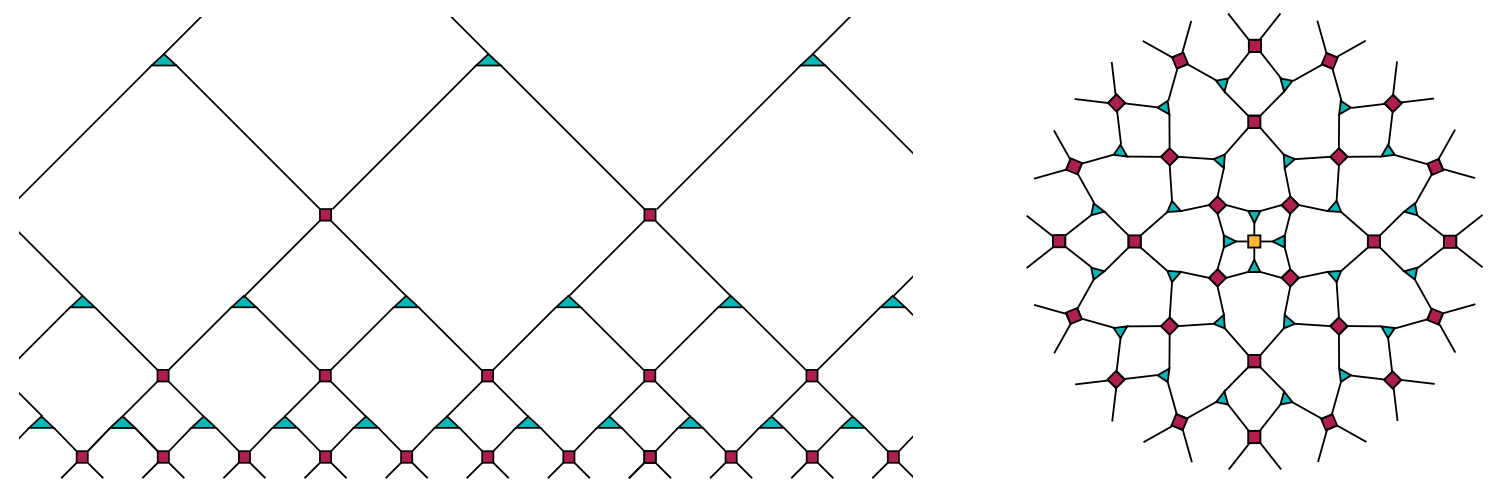

Figure 5. The MERA lattices for states on a line and a circle.

one layer higher takes the wavefunction at scale $\mu$ to the wavefuction at the coarser scale $2 \mu$. More generally, cutting the network in an inhomogeneous way can be understood as enacting a local scale transformation [43]. In this way, the hierarchical structure of MERA encodes an iterative application of local coarse-graining transformations. To understand the rationale underlying the MERA ansatz, it is useful to examine a single layer of the network and ask how it is intended to coarse-grain the wavefunction.

Disentanglers and isometries. A layer of MERA consists of two types of tensors laid out in two rows. The tensors with four legs are called disentanglers. They are $\chi^{2} \times$ $\chi^{2}$ unitary transformations, which select bases wherein incoming UV degrees of freedom will appear locally unentangled. This change of basis is performed in order to prevent UV entanglement from accumulating in the IR wavefunctions defined on higher cuts. In this way, through the action of disentanglers, the MERA network partitions entanglement entropies of intervals into scale-specific contributions.

The second component in every layer of MERA is a row of isometries. These project the locally disentangled UV degrees of freedom into the effective IR Hilbert space. This transformation is isometric, which means that it can be extended to a unitary map

$$
\mathcal{H}_{\mathrm{UV}} \rightarrow \mathcal{H}_{\mathrm{IR}} \otimes \mathcal{H}_{\text {frozen }}
$$

with $\mathcal{H}_{\text {frozen }}$ not participating in further steps of the renormalization $[28,39]$. Our diagrams ignore $\mathcal{H}_{\text {frozen }}$, showing isometries as maps from two UV lines (a $\chi^{2}$-dimensional vector space) to a single IR line (a $\chi$-dimensional vector space.)

Of course, not every wavefunction can be prepared with this ansatz. This is the price we pay for efficiency - by varying the tensors in this fixed network, we scan an $\mathcal{O}\left(\chi^{4} N\right)$ dimensional corner of the full Hilbert space, which we hope includes the ground state wavefunction. This hope has been validated in numerous computations, with the optimized MERA (the state of lowest energy in the variational class [48]) correctly reproducing the spectrum and OPE coefficients of $\mathrm{CFT}_{2}$ s such as the critical Ising model $[47,49,50]$. 


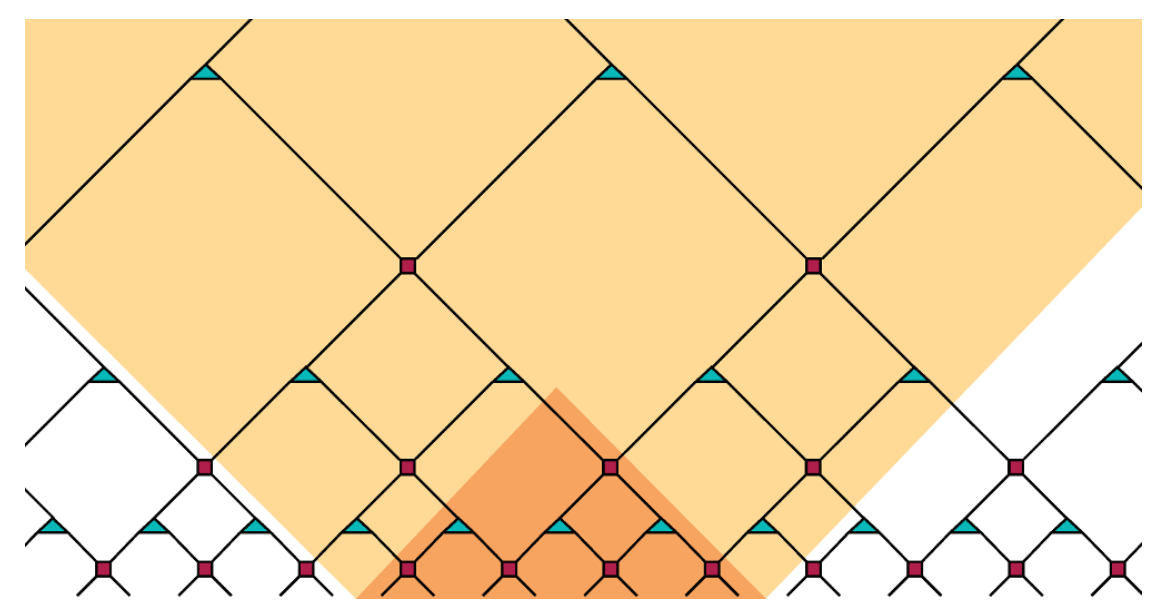

Figure 6. The MERA causal structure. The inclusive causal cone of eight terminal sites is indicated by the expanding yellow region of the network. The exclusive causal cone of the same eight UV sites is indicated by the contracting orange region of the network. The tensors in this region perform a change of basis, which takes the state living on the five sites on the lightlike cut to the eight sites in the UV.

\subsubsection{Causal structure}

This fundamental feature of the MERA network, noticed and exploited already in the initial papers on the subject $[28,49]$, offers the first hint of a relation to kinematic space. A prescient proposal relating MERA to de Sitter space appeared in [29].

Consider the reduced density matrix of an interval $\mathcal{I}$ in a pure state $|\Psi\rangle$ :

$$
\rho_{\mathcal{I}}=\operatorname{Tr}_{\mathcal{I}^{c}}|\Psi\rangle\langle\Psi|
$$

In the language of MERA, we compute it by putting together the tensor network representations of the bra and ket states and joining (tracing out) indices not contained in $\mathcal{I}$. Tracing out these indices means that disentanglers from the $|\Psi\rangle$ network get contracted with their hermitian conjugates from the $\langle\Psi|$ network and cancel out (compare with figure 4(c)). A similar cancellation occurs in the isometries above them, then in the next row of disentanglers, and so on. The ensuing cascade of cancellations divides the network into two parts: the region that determines $\rho_{\mathcal{I}}$, and the region that drops out from it. In analogy with the propagation of signals in a Lorentzian spacetime, we call the former region the 'inclusive causal cone' of interval $\mathcal{I}$.

We shall see in a moment that this notion of 'causality' is the same as in section 2.1.3. Before explaining this, let us consider $\rho_{\mathcal{I}^{c}}$, the reduced density matrix of the complement of $\mathcal{I}$. It too splits up the MERA network into two regions - the inclusive causal cone of $\mathcal{I}^{c}$ and the rest. Altogether, the division of the Hilbert space into localized tensor factors

$$
\mathcal{H}=\mathcal{H}_{\mathcal{I}} \otimes \mathcal{H}_{\mathcal{I}^{c}}
$$

partitions the MERA network into three components: a region that only affects $\mathcal{I}$, an analogous region for $\mathcal{I}^{c}$, and a region that affects the reduced states of both. This division 


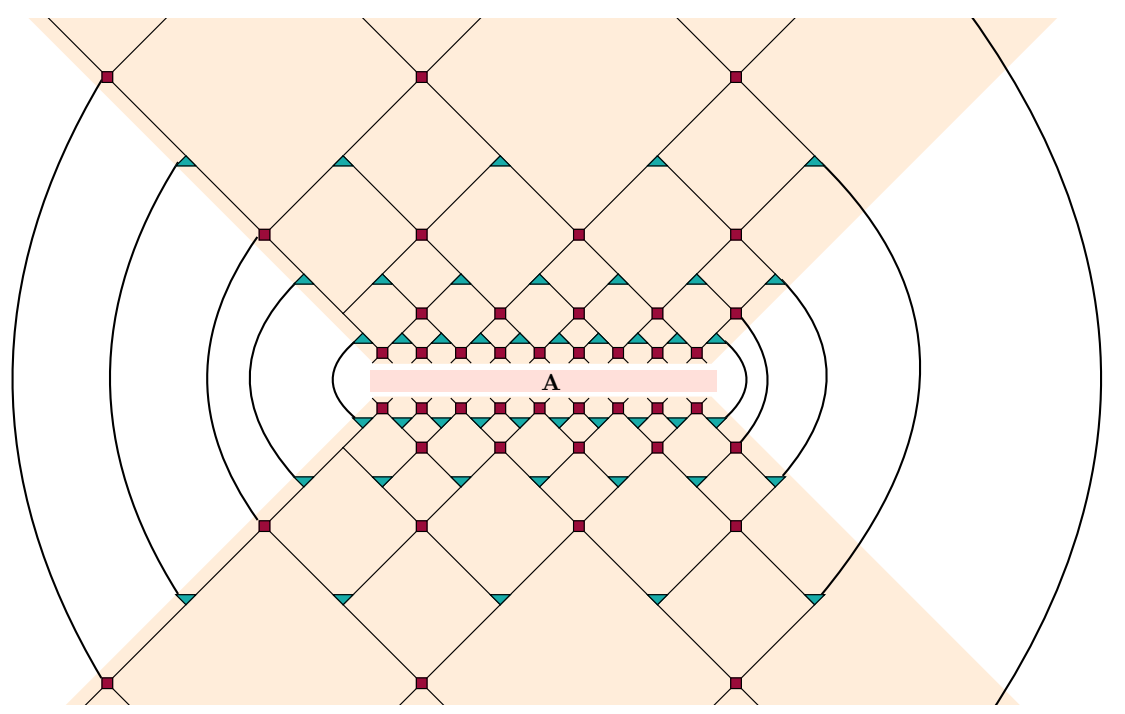

Figure 7. The reduced density matrix $\rho_{A}$ for an interval $A$ in the vacuum can be represented as a tensor network. This network is obtained by taking two copies of MERA, then tracing over $A^{c}$. This causes a cascade of cancellations of disentanglers and isometries. The remaining tensors are only those in the inclusive causal cone of $A$.

is shown in figure 6. For obvious reasons, the first two regions are often called the exclusive causal cones of their respective intervals.

Lightlike coordinates. To keep the nomenclature consistent, we ought to call the boundaries of the aforementioned regions 'lightlike.' These lightlike directions - one leftgoing and one right-going - are linear combinations of the two axes of MERA:

$$
\text { lightlike }=\text { location } \pm \text { scale } \leftrightarrow \quad u, v \text { from eq. (2.5) . }
$$

As in kinematic space, it is convenient to use them as coordinates on MERA. Doing so canonically assigns an interval to every tensor in the network. Specifically, a tensor at lightlike coordinates $u$ and $v$ is the topmost component of the exclusive causal cone of interval $(u, v)$. Notice that the notion of causality defined by eq. (2.20) in MERA is exactly the same as that in section 2.1.3: if a tensor at $\left(u_{2}, v_{2}\right)$ is in the MERA-future (past) of the tensor at $\left(u_{1}, v_{1}\right)$, the corresponding interval contains (is contained in) its counterpart.

The privileged role of the lightlike directions in MERA is a consequence of working with unitary tensors; without unitarity, cancellations discussed below eq. (2.18) would not occur and all parts of the network would affect $\rho_{\mathcal{I}}$ and $\rho_{\mathcal{I}^{c}}$. This marriage of unitarity and causality is displayed by the exclusive causal cones of intervals, whose role amounts to a change of basis. Observe that the action of tensors in the exclusive causal cone of $\mathcal{I}$ is undetectable by observables in $\mathcal{I}^{c}$, so it is a transformation within $\mathcal{H}_{\mathcal{I}}$. After concatenating with the exclusive causal cone, the rather abstract state defined on its lightlike edges is mapped into a local basis of $\mathcal{H}_{\mathcal{I}}$. Although the linear map effected by the exclusive causal cone is an isometric embedding of a smaller Hilbert space in a larger one, when the frozen degrees of freedom from eq. (2.17) are taken into account, it is manifestly unitary. 


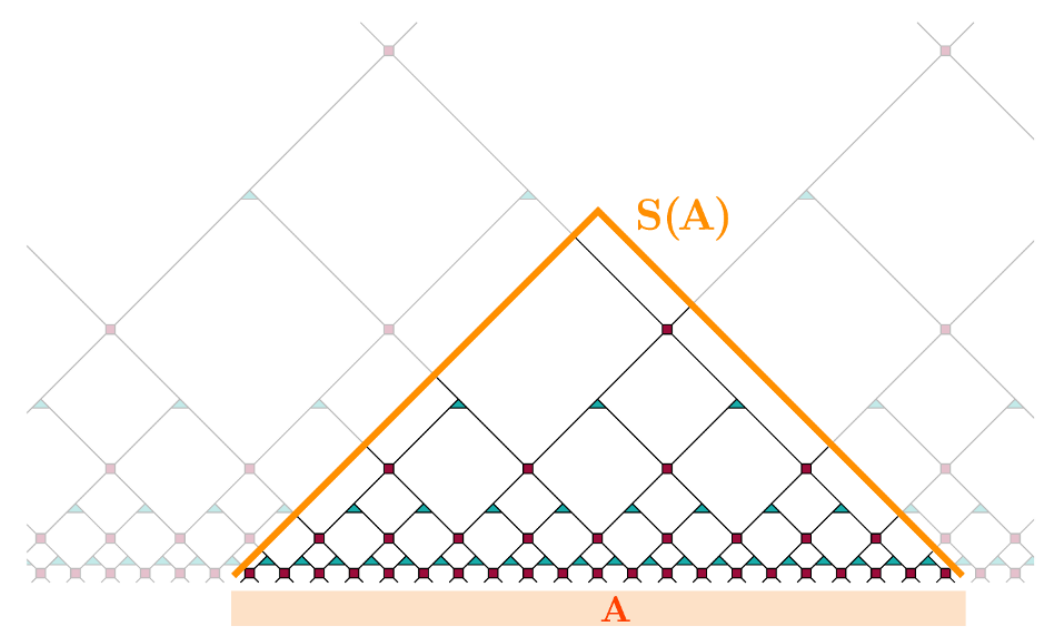

Figure 8. Up to an overall coefficient, the entanglement entropy of a CFT interval $A$ can be estimated by the number of network lines crossing the exclusive causal cone of $A$ times the logarithm of the bond dimension $\log \chi$.

\subsubsection{Entanglement entropies from cut-counting}

A central motif of the present work - and one that motivated holographers' initial interest in MERA [35] - is the simple way the network encodes entanglement entropies. For intervals of less than half system size, a good estimate is obtained by counting the number of lines emanating from the exclusive causal cone of the interval. If each line is counted with weight $\log \chi$, this amounts to computing the logarithm of the dimension of the Hilbert space living on the edge of the exclusive causal cone; see figure 8. In what follows, we will refer to this edge as the 'causal cut,' though the term 'minimal curve' has been used in prior literature [35, 36].

On the one hand, the cut-counting prescription gives a manifest upper bound on the entanglement entropy. We saw in section 2.2.3 that the spectrum of the reduced density matrix of the interval is prepared above the causal cut. The tensors below the cut merely choose a basis in which the state is expressed and therefore have no effect on the entanglement entropy. The maximal value of the entanglement entropy is the logarithm of the dimension of the Hilbert space, in which the state prepared by the network lives. This is precisely what the cut-counting prescription computes.

Though there is no similar argument bounding the entanglement entropy from below, in practice counting cuts gives a good estimate of the entanglement entropy up to a multiplicative constant. In the vacuum, we can surmise this from the logarithmic scaling of entanglement entropy with interval size [51], which agrees with the number of cuts. This heuristic reasoning was verified numerically in [27, 28, 49]. More formal evidence was given in [43], which confirmed that the state on the exclusive causal cones of complementary semi-infinite lines has a thermal entaglement spectrum (see section 4 for a more detailed summary). In light of this fact, the proportionality of entanglement entropy and the number of cuts is equivalent to the extensivity of thermal entropy. For any state built from 
networks with suitably generic tensors, the proportionality of entanglement entropy and the number of cuts was established in [33], which drew on Page's theorem [52].

In the present discussion of the MERA network, we treat the cut-counting prescription as an empirical fact. The reader should remember, however, that no fundamental principle protects this relation and it cannot be expected to hold in full generality. A case in point [53] is the minimally updated MERA network [54], which models a CFT in the presence of an impurity. In such circumstances, any connection between MERA and holography will involve the incremental entanglement entropy per bond instead of a naïve count of bonds.

\section{MERA and kinematic geometry}

Kinematic space encodes the data about CFT subsystems in an elegant geometric way. CFT intervals are organized by location and scale in a Lorentzian space whose metric structure is supplied by conditional mutual information. An analogous representation of CFT subsystem data is given by the MERA network whose tensors are canonically associated with contiguous collections of UV sites. In this section, we outline a series of commonalities that motivate the identification of the two structures. In particular, we propose to view MERA as a discrete counterpart of kinematic space.

Our proposal to associate the MERA network with kinematic space runs contrary to a long-held belief that MERA ought to discretize a spatial slice of the bulk geometry. This idea, first put forward by Swingle [35, 36], gave the impetus to the prolific program of investigating tensor networks vis-à-vis holographic duality [31-34, 37-42], of which the present paper is a part. It is, thus, worthwhile to contrast our novel kinematic proposal with 'the traditional view' of MERA as a discretized spatial geometry. In the discussion to follow, we comment on the conceptual drawbacks of a direct connection to the bulk, which are manifestly absent from the kinematic space perspective. ${ }^{3}$

\subsection{Partial order of MERA and kinematic causality}

The space of geodesics is a partially ordered set. This is an intrinsic property of kinematic space that follows from the containment relation of their boundary support - a property that is invariant under symmetry transformations. The signature of the kinematic metric is the geometric reflection of this structure.

The same applies to MERA: the tensors in the network are partially ordered with respect to their domains of influence. The locality of the tensor contractions, which is built into the skeleton of the network, makes each tensor capable of affecting only a subset of the spatial degrees of freedom. This immediately induces a hierarchy among them in that the regions affected by certain tensors are strictly enclosed within the domain of other tensors' influence. This property of MERA makes no reference to a UV cutoff. Moreover, the unitarity of the tensors promotes this ordering to a true notion of causality: not only do individual tensors affect the state of well-defined spatial intervals, but also the state on

\footnotetext{
${ }^{3}$ Our arguments are structural in character and differ fundamentally from the reasoning followed in [55], which was based on counting degrees of freedom.
} 

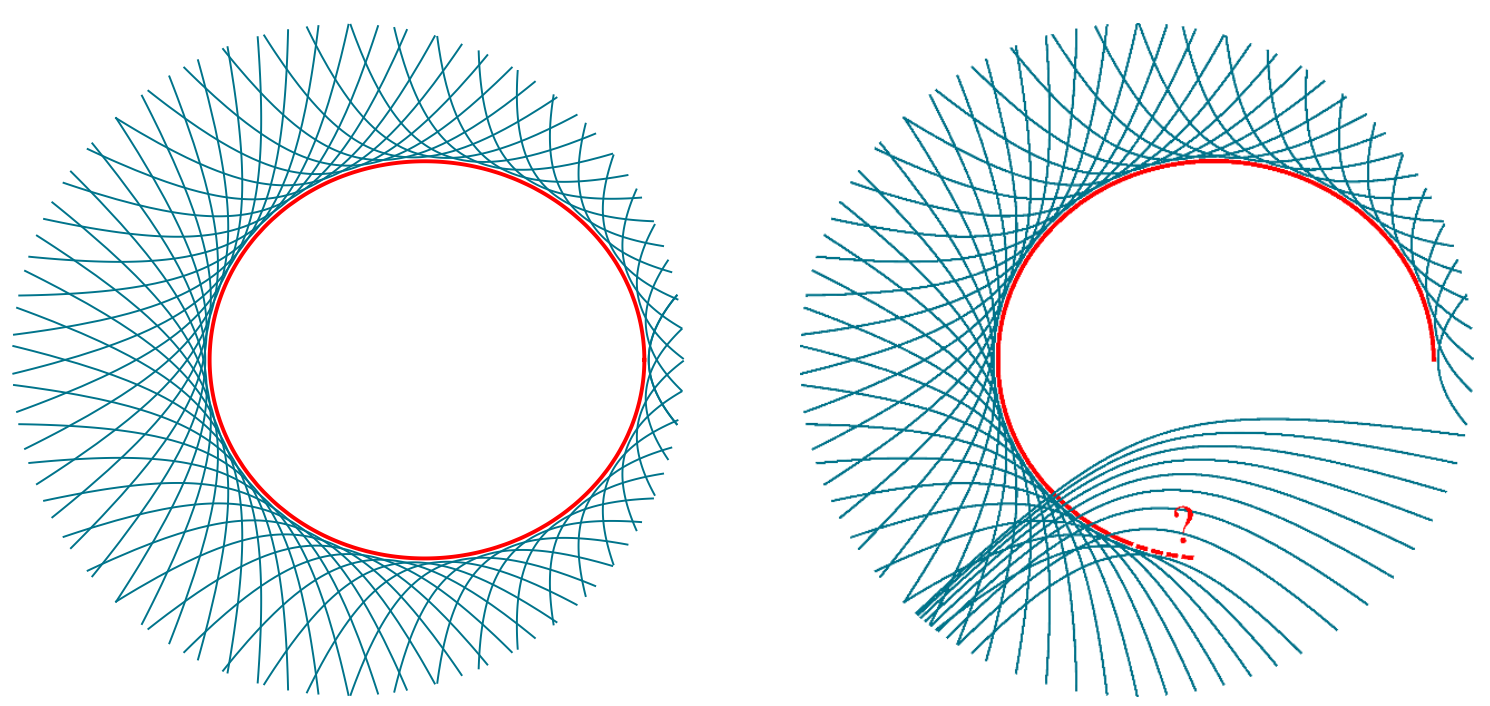

Figure 9. Spacelike (left) and timelike (right) curves in kinematic space as seen from the bulk point of view.

given intervals is influenced only by specific network subregions. We can, therefore, draw light-like directions which restrict the propagation of information in the network.

We observe that the two notions of causality - network and kinematic - coincide. This structure is absent from the hyperbolic plane, all points in which are treated on equal footing. Only upon introducing a cutoff can points on $\mathbb{H}_{2}$ be partially ordered with reference to their distance from the boundary. We shall see that this structural difference has interesting consequences.

\subsubsection{Spacelike versus timelike paths}

An immediate consequence of the Lorentzian signature of kinematic space is a qualitative distinction between kinematic paths that are spacelike, null or timelike. This classification is robust under the action of symmetries and suggests that only certain types of curves, i.e. spacelike, can be used as good kinematic cutoff surfaces. The stipulation that cutoffs must not be timelike is evident in the holographic view of kinematic space. The reason is illustrated in figure 9. A spacelike kinematic trajectory selects a family of geodesics, which has a well-defined outer envelope in the bulk. This envelope acts as a (diffeomorphism invariant) cutoff surface in the spatial geometry. But whenever a trajectory in kinematic space becomes timelike, the bulk cutoff surface is no longer defined [56].

Tensor networks, on the other hand, prepare wavefunctions on selected cutoff surfaces in the network. The causal structure of MERA, however, makes some cutoffs inadmissible: the cut on which the state is defined must be, like in kinematic space, piecewise spacelike or lightlike and never timelike [43]. A MERA network ending on a locally timelike cut cannot be associated to a coarse-grained version of the vacuum wavefunction. The failure of MERA to prepare the wavefunction on a timelike cutoff surface is analogous to the failure of timelike-separated (in kinematic space) geodesics to form a curve in AdS. 
The Euclidean signature of a spatial AdS slice is compatible with any convex bulk cutoff surface [57]. The mixed signature of MERA is therefore in obvious tension with a direct AdS/MERA connection. This is best seen from the simple example of a cutoff that follows a radial geodesic in $\mathbb{H}_{2}$. Symmetry demands that such a cutoff surface, although geometrically admissible in the bulk, map to a vertical (and therefore timelike) cut in MERA. This conclusion can only be averted by introducing extra assumptions into a putative MERA/AdS correspondence.

\subsubsection{Representation of symmetries}

In studying MERA representations of CFT states, an important question concerns the action of conformal symmetry on the tensor network. Because the choice of a cutoff surface on which the state is defined breaks conformal invariance, the cutoff transforms under the conformal group. In two dimensions, conformal symmetry acts locally and can reset the cutoff to an arbitrary function of position. ${ }^{4}$

Local conformal transformations. The primary focus of the discussion in [43] were local conformal transformations in MERA. A conformally transformed wavefunction was recognized as the state living on an inhomogeneous cut in the network. In this way, conformal maps in MERA are implemented by locally changing the cut on which the wavefunction is defined. Importantly, this operation does not affect the rest of the network away from the UV cut. In particular, the causal structure of the MERA network is fixed and independent of conformal transformations. To summarize, the action of the conformal group in MERA can take a uniform UV cutoff to some other, inhomogeneous cutoff, but without affecting the null directions. Consistency then requires that conformal maps take spacelike cutoffs to other spacelike cutoffs.

Such a constraint is guaranteed when MERA is associated with the kinematic geometry. Interpreted in the bulk, however, this seems to impose an artificial restriction on the set of allowed (or MERA-representable) cutoff surfaces: they can never become approximately radial. Since conformal symmetry transforms radial and other bulk surfaces into one another, such a limitation would be a radical breaking of conformal symmetry.

Inhomogeneity of a causal cut. Causal cuts in MERA are not homogeneous. Their lightlike segments are uniform, but the top of a causal cut where left-going and right-going cuts meet is distinct from the rest. The non-uniform shape of a causal cut in MERA is readily understood in the kinematic interpretation. The top corresponds to the geodesic $g$ supported on the base of the chosen lightcone while other points on a causal cut correspond to narrower geodesics that share one endpoint with $g$ and are otherwise contained within it (compare e.g. figure 1 in [22]). The insensitivity of both the MERA and kinematic partial order to the UV-cutoff ensures that this point will remain special under local conformal maps.

The AdS isometries, on the other hand, map different points on the same geodesic to one another. In other words, geodesics are homogeneous, a fact that forbids special points.

\footnotetext{
${ }^{4}$ Of course these statements hold up to the usual artifacts of discretization.
} 
When identifying the causal cut with an AdS geodesic -as the direct AdS/MERA connection suggests- one might try to assuage this discrepancy by declaring that the special point on a cut in MERA corresponds to some select point on a bulk geodesic, chosen according to some prescription. Any such prescription, however, must refer to a UV cutoff; in the absence of a UV cutoff there is no reference with respect to which a special point may be chosen. Because conformal symmetry acts on the cutoff, it must also affect the choice of a preferred point on a geodesic. Yet in MERA, the top of a causal cut is fixed, its location blind to any changes in the cutoff. This reveals that the conformally invariant notion of causality in MERA disfavors a naïve partial ordering of the hyperbolic plane induced by a UV cutoff. But it is in full agreement with the causal structure of kinematic space, which is likewise conformally invariant.

\subsection{Localization of information}

\subsubsection{Crofton form and volumes in MERA}

In section 2.1.4, we observed that the notion of volume of kinematic space (eq. (2.4)) hails from information theory: it is the conditional mutual information (2.12) of three contiguous intervals. Let us inspect the same quantity in MERA.

Conditional mutual information localizes in MERA. When we apply the cutcounting prescription reviewed in section 2.2 .4 to

$$
I(A, C \mid B)=S(A B)+S(B C)-S(A B C)-S(B),
$$

we obtain figure 10. The cuts associated with the positive terms in (2.12) are in large part the same as the cuts for the negative terms, leading to cancellations. The net result comes from a localized part of the network, whose boundaries are lightlike. In other words, the conditional mutual information of neighboring intervals localizes in a causal diamond. For intervals with endpoints at

$$
A=(u-\Delta u, u) \quad \text { and } \quad B=(u, v) \quad \text { and } \quad C=(v, v+\Delta v),
$$

the relevant causal diamond resides between $u$ and $u-\Delta u$ in the left-moving coordinate and between $v$ and $v+\Delta v$ for the right-moving one.

In the end, the entire MERA network is a tilted chessboard of causal diamonds, each of which computes some conditional mutual information. The grid of lightlike coordinates demarcates conditional mutual informations of different triples of intervals. Figure 11, which displays these facts, is a faithful copy of figure 3, which highlights the analogous characteristics of kinematic space.

What does conditional mutual information count? Figures 10 and 11 give a crisp answer: conditional mutual information counts how many isometries live in the appropriate causal diamond. Eq. (2.12) asks for the net reduction in the number of lines passing through the causal diamond as we go from the bottom up. The only way we can register a net loss of lines is if a line is soaked up by an isometry. Indeed, every isometry accounts for precisely one line, which enters the diamond from the bottom but does not emerge at the top. Counting the decrease in the number of lines is equivalent to counting isometries. 

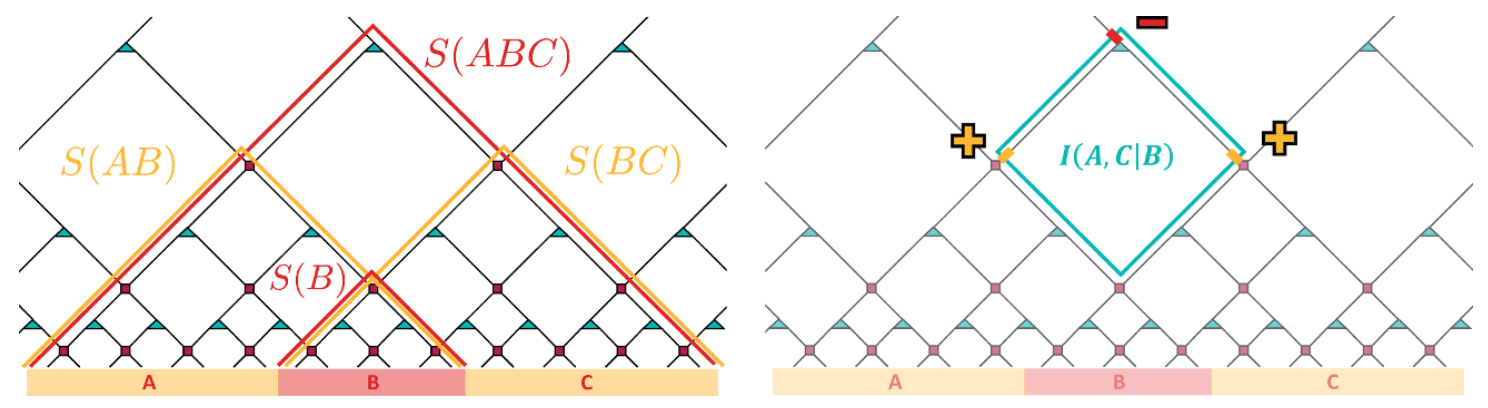

Figure 10. Conditional Mutual Information in MERA. Most cuts that contribute to the computation of $I(A, C \mid B)$ cancel in the alternating sum. The net contribution to the conditional mutual information arises from a localized region of the network.

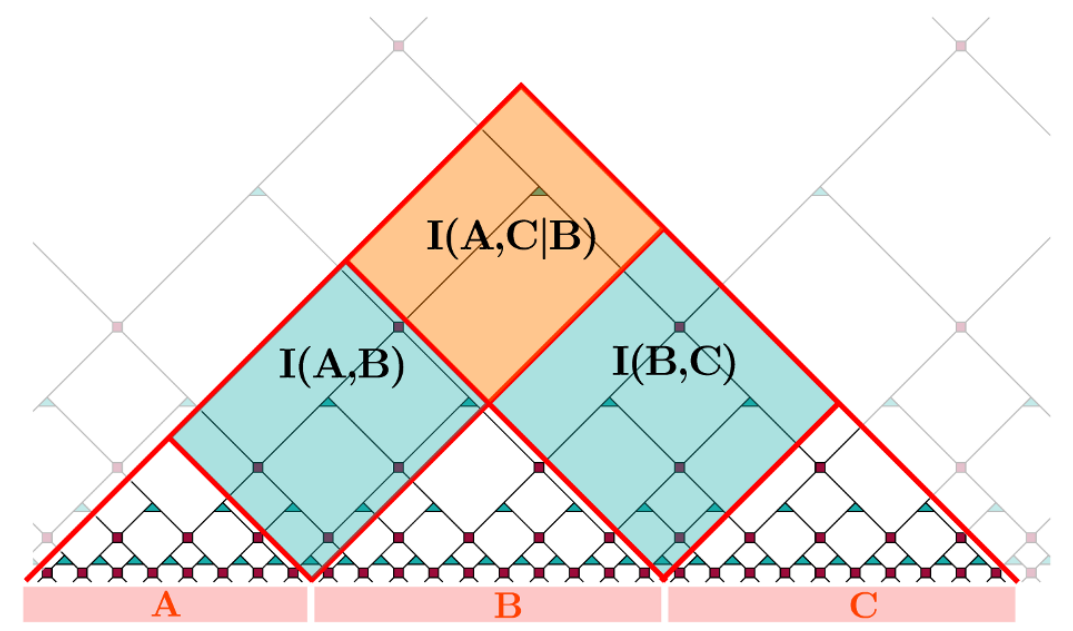

Figure 11. Localization of mutual information in MERA. We indicate the local regions of the network that control the computation of the mutual information of two neighboring intervals, and the conditional mutual information of three neighboring intervals.

Conditional mutual information as volume. We propose to adopt conditional mutual information as a definition of volume in MERA. The two facts highlighted above guarantee that this is a reasonable proposal: conditional mutual information localizes in MERA and counts a crisply defined object - the isometries contained in a causal diamond. In other words, we observe that for $A, B, C$ defined in eq. (3.1):

$$
\mathcal{D}(\text { isometries })=I(A, C \mid B) \text {. }
$$

We declare this quantity a discrete volume form, in analogy to eq. (2.4) in kinematic space.

In the upcoming second part of this work [30], where we discuss our more general compression networks, we will appreciate better the rationale for working with eq. (3.2). The volume of a causal diamond computed by (3.2) evaluates the amount by which the tensors in the diamond compress the state living on its past edges. This is how eq. (3.2) should be viewed in applications beyond the standard MERA. In the special case of the vacuum MERA, this 'density of compression' is directly proportional to a naïve count of 
isometries. We give a short summary of the compression networks in section 5 , referring to [30] for details.

It is worth noting that in the traditional holographic view of MERA the connection between conditional mutual information and localized volumes of the network is puzzling. If we represent the terms in eq. (2.12) by geodesics in the bulk, no such localization occurs. Instead, the calculation involves an extended region in the spatial geometry, which reaches all the way to the asymptotic boundary. When $A$ and $C$ are taken to be small as in eq. (3.1), the bulk region associated with $I(A, C \mid B)$ becomes a fattened geodesic subtending $B$. In MERA this limit shrinks the relevant causal diamond to a small number of tensors. This again motivates relating small regions in MERA to bulk geodesics.

A metric for MERA. In section 2.1.3, we assembled the kinematic metric (2.11) from two ingredients: the causal structure (eq. (2.5)) and the volume form (2.4). In section 2.2.3 we recognized that MERA has an identical causal structure. Now eq. (3.2) gives us a notion of volume, which is a direct analogue of eq. (2.4). These reasons justify conceptualizing MERA as a discrete version of kinematic space. More explicitly, we may write down a discrete tensor network metric

$$
d s_{\text {T.N. }}^{2}=I(\Delta u, \Delta v \mid B) \stackrel{\text { MERA }}{\longrightarrow}(\# \text { isometries }) \Delta u \Delta v
$$

which in the case of the familiar MERA simply counts isometries in causal diamonds. This metric is the obvious counterpart to eq. (2.11) in kinematic space.

Differential entropy and cut-counting in MERA. One attractive feature of kinematic space is that volumes in it reproduce the differential entropy formula [20]; see eq. (2.7). Metric (3.3) ought to give rise to a similar relation in MERA.

Indeed, any spacelike cut across MERA defines a (possibly non-uniform) UV cutoff and a coarse-grained Hilbert space; see section 2.2.2. The logarithm of the dimension of that Hilbert space is proportional to the number of indices living on the cut. Because every line ends on some isometry in the UV part of the network, the logarithm of the dimension of the Hilbert space defined by a cut is equal to the volume of MERA living above that cut, counted according to eq. (3.2). The equality between the 'volume' of a subregion of MERA and the number of lines on its boundary follows from a discrete version of Stokes' theorem.

This argument is an exact analogue of the reasoning articulated below eq. (2.7). Thus, computing the size of a coarse-grained Hilbert space by counting indices on its defining cut is the MERA version of the differential entropy formula. As a special case, this recovers the cut-counting prescription for entanglement entropy, which we revisit in section 3.2.2. More generally, counting cuts assigns an entropic quantity to any (possibly non-uniform) spacelike UV cutoff, which in the bulk is represented by a collection of tangent geodesics (see figure 9).

\subsubsection{Entanglement entropy}

The feature of MERA that makes it especially relevant for holography is the way it geometrizes entanglement entropies. In section 2.2.4 we reviewed the cut-counting prescription in MERA: estimating the entanglement entropy of an interval by counting the lines 


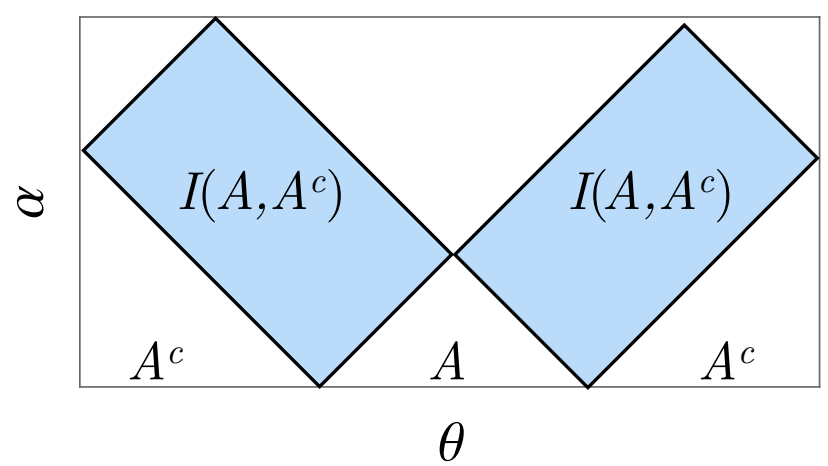

Figure 12. The entanglement entropy of $A$ is given by half of the total kinematic volume of the 'causal wings' depicted in the figure.

which cross the causal cut. This special property of the optimized network was used in section 3.2.1 to place a physical metric on MERA (eq. (3.3)) and recognize it as a faithful representation of the kinematic geometry.

If MERA discretizes the kinematic space, however, the causal cut in MERA becomes a discrete version of the kinematic causal cone. The integral geometric computation of entanglement entropies then ought to be consistent with the cut-counting prescription along this causal cut. Recall that in a pure state, the entanglement entropy of an interval $\mathcal{I}$ is half the mutual information of $\mathcal{I}$ and its complement, $\mathcal{I}^{c}$ :

$$
S(\mathcal{I})=\frac{1}{2} I\left(\mathcal{I}, \mathcal{I}^{c}\right)
$$

As we saw in section 2.1.4, the mutual information of two adjacent intervals can be read off from kinematic space as the volume of a causal diamond, which includes the common endpoint of both intervals. In the case at hand, we actually have two causal diamonds, because $\mathcal{I}$ and $\mathcal{I}^{c}$ have two endpoints in common (see

$$
S(u, v)=\frac{1}{2} \int_{u}^{v} d \tilde{v} \frac{\partial S(u, \tilde{v})}{\partial \tilde{v}}+\frac{1}{2} \int_{v}^{u} d \tilde{u} \frac{\partial S(\tilde{u}, v)}{\partial \tilde{u}}
$$

The two terms in this formula come from the two causal diamonds in figure 12. In the context of MERA, their integrands become densities of lines that cross the causal cut (section 3.2.1). This is precisely what the cut-counting prescription mandates.

To see this more clearly, consult figure 13, which is the MERA analogue of figure 12 . Taking advantage of the localization of mutual information in MERA illustrated in figure 11, we know that the entanglement entropy of $A$ is given by the number of isometries living in the highlighted part of the network. But the same count yields the number of lines crossing the causal cut of $A$. In effect, we are learning that the cut-counting prescription of section 2.2.4 secretly enumerates the isometries responsible for correlating the interval with its complement. This is in direct analogy with the way kinematic space encodes the length of a Ryu-Takayanagi geodesic as the 'number of geodesics' connecting the boundary interval with its complement. The counting of geodesics is done with the Crofton measure, which is a geometric counterpart of the density of isometries in MERA. 

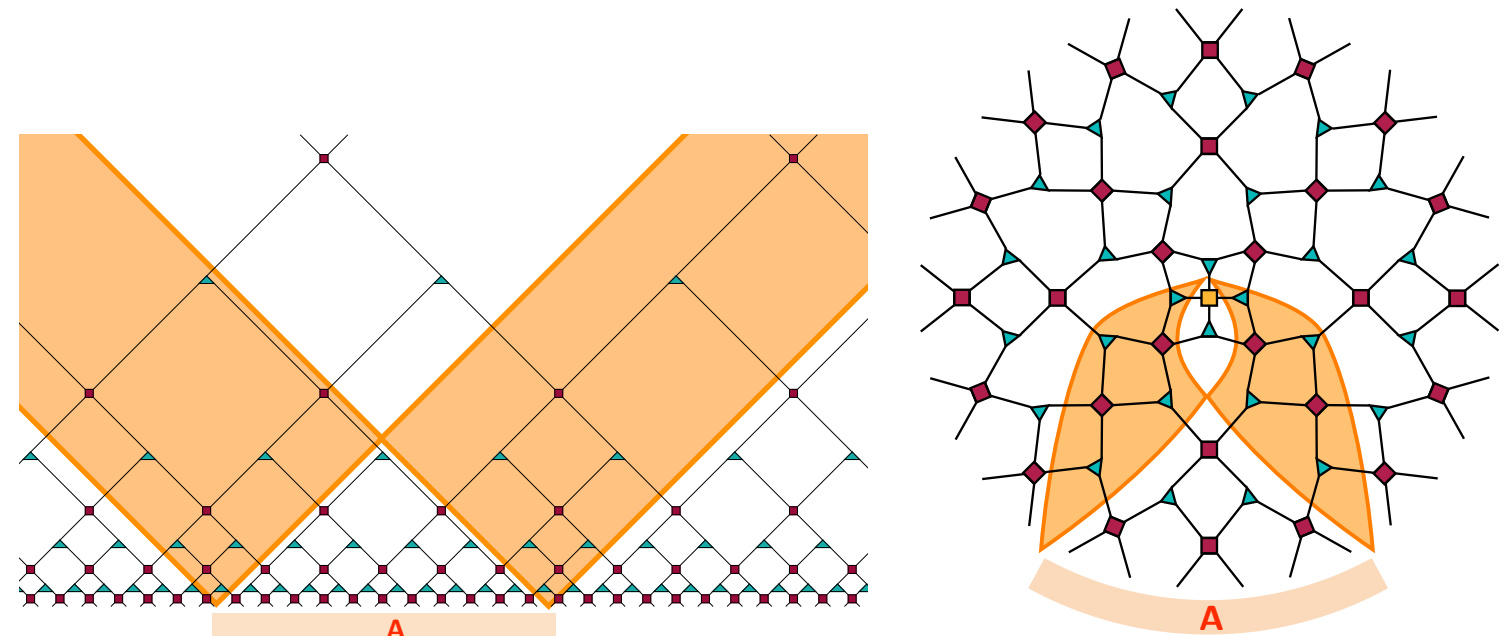

Figure 13. The entanglement entropy of a CFT interval $A$ is computed by the number of network isometries responsible for correlating $A$ with its complement. This is consistent with the integral geometric computation of the corresponding geodesic length (figure 12). An application of the discrete Stokes' theorem recovers the cut-counting prescription of section 2.2.4. The figure shows the network volume relevant for the EE on the line (LEFT) and the circle (RIGHT). It contains all tensors in the inclusive causal cones of the two endpoints of $A$, excluding their intersection. This network region prepares the entanglement spectrum between $A$ and $A^{c}$.

In contrast, if we place the network directly on the time-slice of AdS, the relation between volumes of 'causal wings' and entanglement entropies appears mysterious. It seems to imply that a special region in the bulk — which lacks an independent motivation in the AdS/CFT correspondence - quantifies the correlation between a given interval and its complement in terms of its volume; see figure 13. Insisting on an AdS/MERA correspondence appears to add another peculiar property to its putative dictionary, a peculiarity that is readily resolved by the kinematic proposal.

\subsection{MERA as renormalization}

\subsubsection{Coarse-graining with MERA}

As we reviewed in section 2.2.2, MERA provides a graphical representation of renormalization in real space. The vertical direction corresponds to scale in the field theory. Cutting MERA on different levels defines states, which are related to one another by coarse-graining or fine-graining. As we go higher up in MERA, the successively coarse-grained states live in Hilbert spaces of exponentially decreasing sizes (entropies).

The same features are observed in kinematic space; its identification with the space of CFT intervals makes it a natural domain for real space cutoffs. The two coordinates of kinematic space, $\theta$ and $\alpha$ (see eq. (2.5)) also correspond to location and scale. The role of $\alpha$ as setting a scale is evident from its definition as the half-width of a field theory interval. Cutting off kinematic space at $\alpha=\alpha_{*}$ imposes a real space cutoff - it amounts to declaring $2 \alpha_{*}$ to be the smallest resolution in the field theory. The spatial size of a cutoff 
surface in kinematic space also varies exponentially with the cutoff; in the vacuum on a circle, metric (2.11) expressed in terms of $\theta$ and $\tilde{\rho}=-\log (\csc \alpha+\cot \alpha)$ is:

$$
d s_{\text {kin }}^{2}=\frac{c}{3}\left(-d \tilde{\rho}^{2}+\cosh ^{2} \tilde{\rho} d \theta^{2}\right) .
$$

Holographically, every real space cutoff defined by a curve in kinematic space selects a set of bulk geodesics. These in turn identify a bulk cutoff surface by their outer envelope as we illustrated in figure 9. This proposal for the holographic cutoff has the appealing feature that it is manifestly diffeomorphism invariant, because it is implemented on bulk geodesics that make no reference to AdS coordinate systems. Interestingly, the kinematic cutoff can be further promoted to a precise coarse-graining prescription for CFT operators, which exploits the structure of the operator product expansion [11]. We comment further on the relevance of [11] to MERA in the Discussion.

On a spatial slice of the bulk geometry, the radial direction $\rho$ is also dual to changes of scale in the CFT [58-60]. Regulating large scale divergences on the gravity side with a radial cutoff $\rho=\rho_{*}$ is dual to selecting an ultraviolet cutoff in the CFT. When we push the radial cutoff $\rho_{*}$ to infinity, the area of the cutoff surface grows exponentially. This is captured by the spatial metric:

$$
d s^{2}=L^{2}\left(d \rho^{2}+\sinh ^{2} \rho d \theta^{2}\right)
$$

The interpretation of MERA as a real space RG transformation, however, can be leveraged to distinguish between the two types of geometric coarse-graining suggested above. As we explain in the next section, the constraints that causality imposes on the RG operation of MERA act in favor of the kinematic proposal.

\subsubsection{Real space RG and causal cuts}

Consider two points on the 1-D boundary slice where the CFT state lives. Any such choice splits the CFT into two regions: an interval $A$ and its complement $A^{c}$. In a pure state such as the vacuum the entanglement entropies $S(A)$ and $S\left(A^{c}\right)$ are equal. This fact is nicely captured by the Ryu-Takayangi proposal: a unique minimal geodesic homologous to both $A$ and $A^{c}$ joins the two boundary points.

In MERA, for any selection of two spatial points there are two distinct causal cuts in the network, which bound the exclusive causal cones of $A$ and $A^{c}$, respectively (see figure 14). The two cuts typically do not cross the same number of links; only the minimal one has the correct count of links to match the entanglement entropy. Nevertheless, both lightcones are physically meaningful. In the view of MERA as a real space RG transformation, every local application of disentanglers and isometries performs a local coarse-graining of the wavefunction. Such coarse-grainings can be understood as a change of basis, but only if the cutoff surface is piecewise spacelike or null at every RG step. In this way, we obtain an upper bound for the allowed local coarse-graining of an interval. The two causal cuts encode the maximally coarse-grained state of $A$ and $A^{c}$, respectively.

To appreciate the importance of the two distinct MERA lightcones in a different way, observe that the tensors contained in the exclusive causal cone of an interval build local 

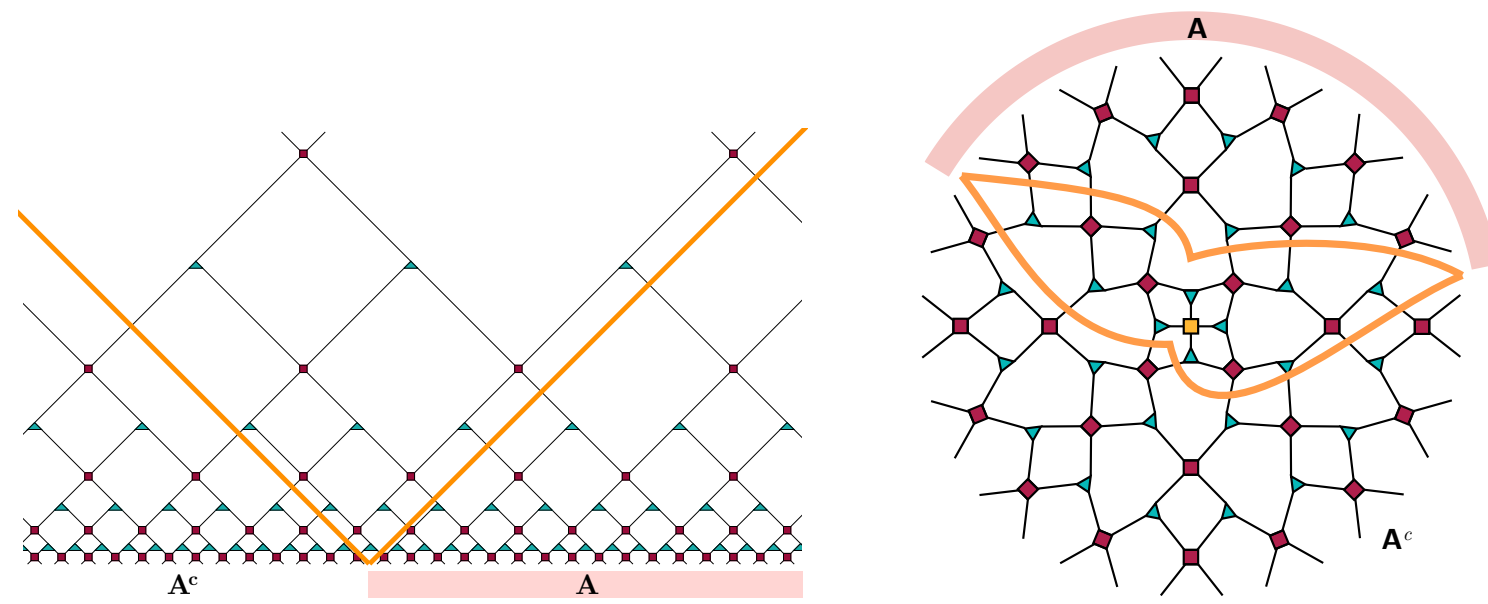

Figure 14. Causal cuts for a region $A$ and its complement $A^{c}$. For both MERA on the line and circle, the causal cuts for $A$ and its complement $A^{c}$ are distinct.

correlations among the interval's lattice sites. This amounts to selecting a local basis for representing the state and leaves the entanglement spectrum unaffected. In other words, the entanglement spectrum of $A$ is solely prepared in the region between the two causal cones.

This feature of MERA is naturally included in the kinematic proposal: the two complementary intervals possess distinct causal cones, each of which bounds the set of bulk geodesics anchored on the respective boundary region. There exists, moreover, a finite 'volume' of geodesics that connect the two intervals, a fact reflected by the separation of the two lightcones in kinematic space. When approached from the traditional AdS/MERA perspective, however, no meaningful geometric counterpart exists for the non-minimal causal cut. This contradicts the equal treatment of the two cuts in the network and seems to select a peculiar, IR-probing curve associated to the coarse-grained state of the larger interval. By the AdS/MERA interpretation, that coarse-grained state should have been instead associated to the minimal geodesic.

\section{MERA for boundary gravitons and two-sided black holes}

\subsection{Boundary gravitons}

Thus far, we have argued for identifying the vacuum MERA with the kinematic space of a time slice of pure $\mathrm{AdS}_{3}$. This conclusion automatically extends to conformal descendants of the vacuum - states related to the vacuum by a local conformal transformation. In MERA, wavefunctions of such states can be read off from inhomogeneous UV cuts [43]. In particular, going from the vacuum to a descendant does not change local properties of the network. On the bulk side, descendant states are represented by so-called boundary gravitons [61]. They are locally $\mathrm{AdS}_{3}$ geometries, which differ from global $\mathrm{AdS}_{3}$ by large diffeomorphisms. Importantly, a large diffeomorphism changes lengths of geodesics by two additive pieces, which carry no joint dependence on the two endpoints [62, 63]:

$$
S(u, v) \rightarrow S(u, v)+\Delta \mu(u)+\Delta \mu(v)
$$




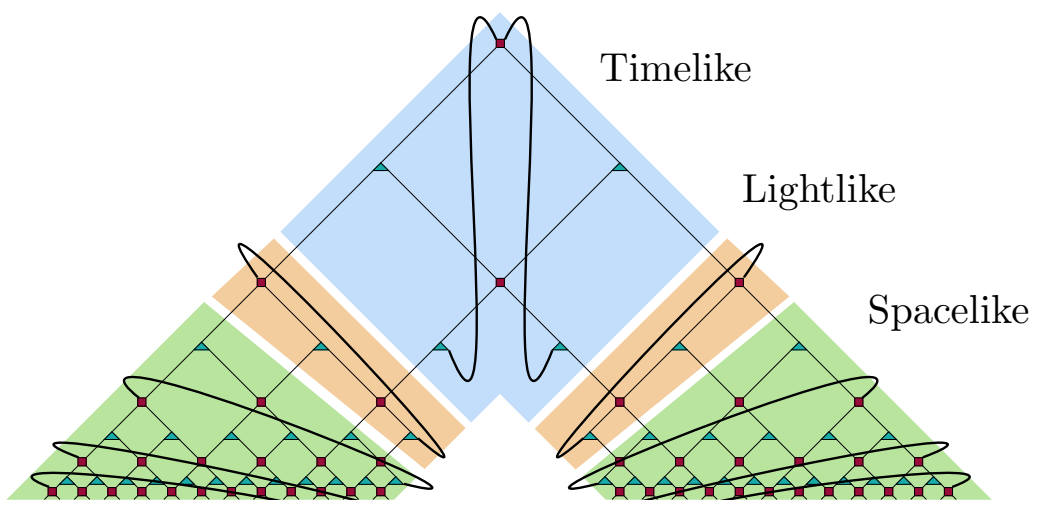

Figure 15. The quotient of the optimized vacuum MERA, which prepares the thermofield double state. In different parts of the network the identifications act in a timelike, lightlike and spacelike manner, respectively. The network displayed here identifies lines that are $k=2$ layers apart, so $s=2^{k}=2^{2}$ in eq. (4.7). The figure is reproduced with permission from [43].

This change leaves kinematic volumes (2.4) invariant. We reach the same conclusion by noting that a boundary conformal transformation that preserves a time slice of the CFT maps $x \rightarrow \tilde{x}=f(x)$. Applying this transformation to $u$ and $v$ in the kinematic metric (2.11) gives:

$$
d s_{\text {kin }}^{2}=\frac{\partial^{2} S(u, v)}{\partial u \partial v} d u d v=\frac{\partial^{2} S(\tilde{u}, \tilde{v})}{\partial \tilde{u} \partial \tilde{v}} d \tilde{u} d \tilde{v}
$$

This illustrates that the kinematic space defined in eq. (2.11) is invariant under all conformal transformations which preserve a time slice of the CFT. The only dependence on the conformal frame is introduced by the UV cutoff.

\subsection{The thermofield double state and the two-sided BTZ black hole}

The thermofield double state in the CFT and in MERA. For a non-trivial application of local conformal transformations, consider a map that acts not on the full line $\mathbb{R}$, but the line minus a point, $\mathbb{R} \backslash\{0\}$. The two semi-infinite lines on either side of the excluded point can each be mapped to an infinite line by the logarithmic map:

$$
x \rightarrow(\beta / 2 \pi) \log |x|
$$

In this way, we view the vacuum on $\mathbb{R}$ as an entangled state on $\mathbb{R} \times \mathbb{R}$. Famously, in a conformal field theory this entangled state is the thermofield double state. One may further quotient the two $\mathbb{R}$ s by a discrete translation $\log x \sim \log x+\log s$ to obtain the thermofield double state on $S^{1} \times S^{1}$. The circumference of the $S^{1}$ sets a natural scale, in which to express the otherwise dimensionless inverse temperature:

$$
(\beta / 2 \pi) \log s \equiv 2 \pi L
$$

In ref. [43] our collaborators and we performed these operations in the optimized MERA network. The conformal transformation (4.3) was enacted by cutting the network along the two null rays emanating from $x=0$. After the cut, the quotient identifies identical 
pieces of the causal cone of the origin. Alternatively, we can apply the quotient prior to the conformal map (4.3). This produces the entire network shown in figure 15, including the regions living below the null rays. In this view, the two semi-infinite lines are modded out by a discrete scaling transformation $x \sim s x$.

The BTZ black hole as a quotient of $\mathbf{A d S}_{\mathbf{3}}$. The field theory operations outlined above reflect a famous fact in 3-d gravity: that the two-sided BTZ black hole is a quotient of pure anti-de Sitter space [64]. Consider the Poincaré-AdS $\mathrm{Ad}_{3}$ metric restricted to $t=0$ :

$$
d s^{2}=\frac{d x^{2}+d z^{2}}{z^{2}}
$$

In order to quotient $\mathbb{R}_{-}$and $\mathbb{R}_{+}$by a discrete scale transformation, select a family of geodesics centered at $x=0$ whose radii are related by powers of $s$ :

$$
x^{2}+z^{2}=s^{2 n} r^{2} \quad \text { where } n \in \mathbb{Z}
$$

Identifying these geodesics with one another produces a topological cylinder, which is the static slice of the two-sided BTZ geometry; see figure 16. This identification can be canonically extended away from the time slice to produce the full, 2+1-dimensional BTZ spacetime with two asymptotic regions. The inverse temperature of the black hole in units of the $\mathrm{AdS}_{3}$ curvature scale is given by eq. (4.4):

$$
\beta / L=4 \pi^{2} / \log s
$$

In the limit $n \rightarrow-\infty$, the geodesics in figure 16 zoom on a point on the boundary at $x=0$. This location, which is a fixed point of the quotiented discrete scale transformation, separates the two semi-infinite lines into which the $x$-axis decomposes under map (4.3). After the quotient, every fundamental domain in $x>0$ represents a copy of one asymptotic boundary while fundamental domains in $x<0$ are copies of the other asymptotic boundary. In the bulk, the line $x=0$ is also meaningful. It connects points of closest approach of identified geodesics and, therefore, comprises images of the bifurcation horizon. Many good reviews of these facts exist, including [65].

\subsection{The quotient MERA is the kinematic space of the two-sided black hole}

Let us compare the tensor network shown in figure 15 with the space of geodesics on a static slice of the two-sided BTZ black hole. Due to the discrete nature of the tensor network we may only quotient MERA by discrete scalings with $s=2^{k}$ for $k \in \mathbb{Z}_{+}$.

Structure of the identifications. Observe that in the tensor network quotient in figure 15 not all identifications of indices are on the same footing. In the middle of the network we connect lines which are timelike-separated in the MERA sense. In the UV the identification joins indices that are spacelike-separated. The network contains two such regions, one on each side of the thermofield double. Separating the spacelike-identified regions from the timelike-identified one are single lines of tensors, which after the quotient form closed lightlike curves. These distinct components of the thermofield double MERA correspond to 

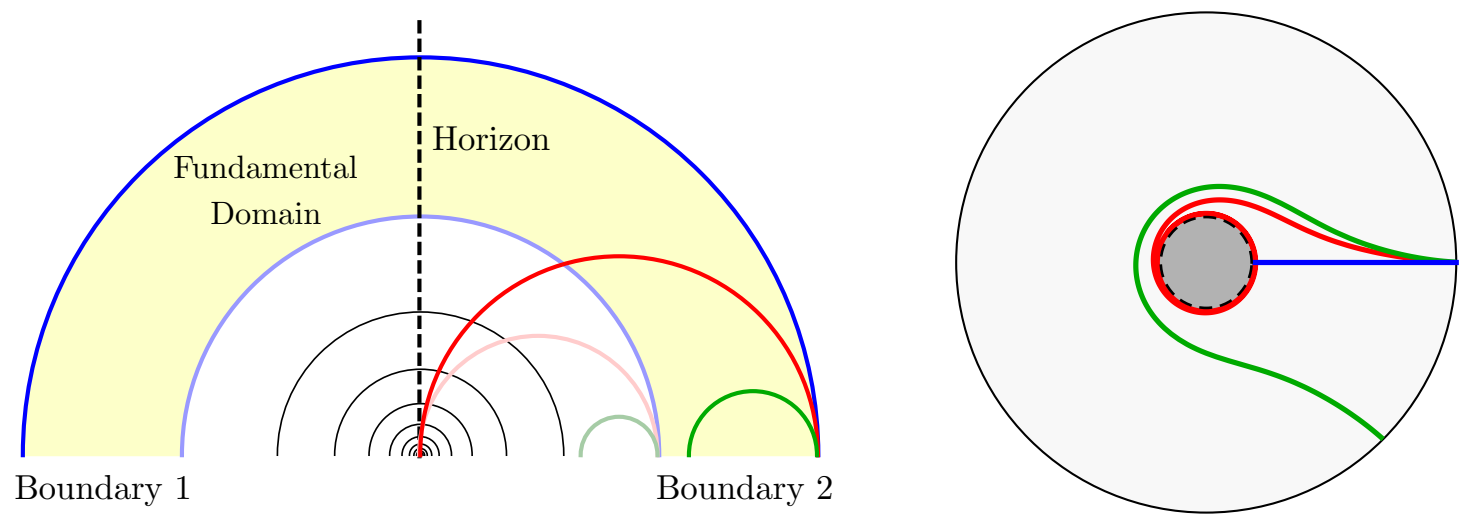

Figure 16. Identifying concentric geodesics on a static slice of $\mathrm{AdS}_{3}$ produces a static slice of the two-sided BTZ black hole. Three BTZ geodesics are shown, along with the $\mathrm{AdS}_{3}$ geodesics from which they descend. The green spacelike-identified geodesic is anchored on a single boundary. The red lightlike-identified geodesic circles the horizon indefinitely. The blue timelike-identified geodesic connects the two sides.

analogously distinguished classes of geodesics in the two-sided BTZ geometry. ${ }^{5}$ We marked the three classes in figure 16.

The first of these are the timelike-identified geodesics. A canonical example of these are the geodesics in eq. (4.6), which define the geometric quotient in figure 16. More generally, a geodesic becomes identified with a timelike-separated image of itself if one of its endpoints is negative $(u<0)$ while the other one is positive $(v>0)$. This means that timelike-identified geodesics connect opposite sides of the two-sided black hole. They are horizon-crossing geodesics.

The spacelike-identified geodesics remain on one side of the horizon. Their endpoints are either both positive $(0<u<v)$ or both negative $(u<v<0)$. In the bulk, such geodesics do not reach the horizon.

The marginal case separating the previous two are lightlike-identified geodesics. Recall that $u$ and $v$, the left and right endpoint of a geodesic, are lightlike coordinates in kinematic space. Thus, the geodesic $(u, v)$ is lightlike-separated from its scaled image $(s u, s v)$ if and only if $u=s u=0$ or $v=s v=0$. This is consistent with the scope of the timelike-identified $(u<0<v)$ and spacelike-identified regions $(u<v<0$ and $0<u<v)$.

The lightlike-identified geodesics are the borderline case, which separates horizoncrossing geodesics from those which remain a finite distance apart from the horizon. They are tangent to the horizon. A boundary-anchored geodesic can only become tangent to the horizon after spiraling around it infinitely many times. The infinite winding of the lightlike-identified geodesics can be seen in figure 16. In the covering space, such geodesics cross infinitely many copies of one type of asymptotic boundary. For a more extensive discussion of infinitely winding geodesics in the BTZ geometry, consult [23].

Timelike-identified regions. In ref. [43], our co-authors and we explained that the timelike-identified region of the quotient network prepares the spectrum of the thermofield

\footnotetext{
${ }^{5}$ They are also reminiscent of the 'torus with whiskers' analyzed in [66].
} 


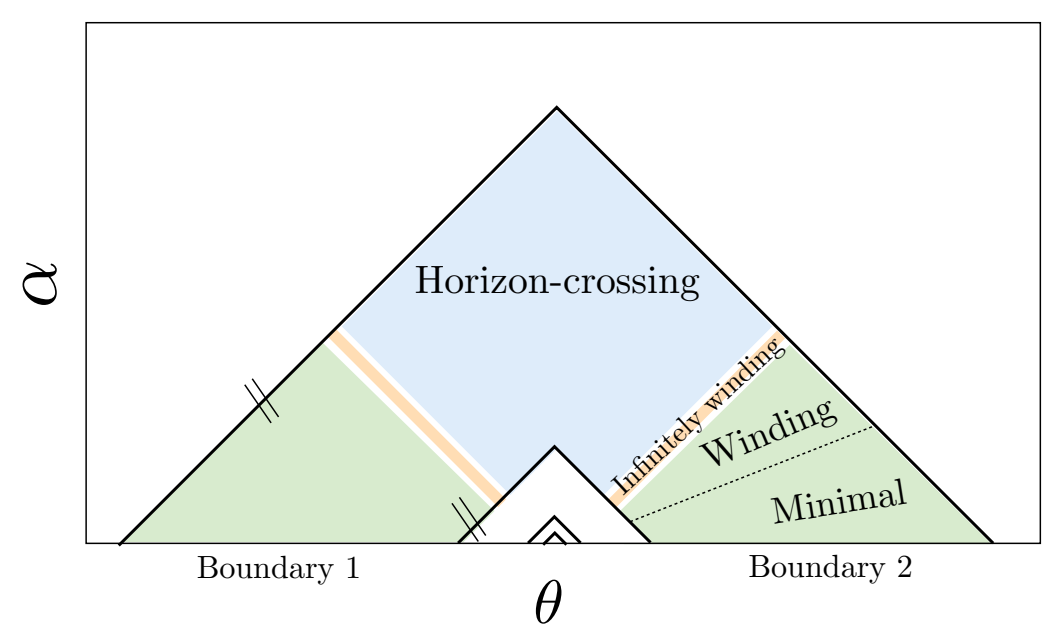

Figure 17. The kinematic space for the two-sided BTZ black hole, to be compared with figures 15,16 . The BTZ kinematic space is obtained as a quotient of the vacuum kinematic space, where two causal cuts are identified. A fundamental domain is labeled above, which separates into horizon-crossing, winding (entwinement), and minimal (entropy) geodesics. The points on the lightlike lines indicated correspond to infinitely winding geodesics.

double state. In other words, this region is solely responsible for fixing the correlations between the two sides. This is exactly what we expect from the kinematic interpretation of MERA, which relates this region of the network to geodesics that cross the horizon and connect the two asymptotic boundaries. As an example, such geodesics were used to compute two-sided correlators in the thermofield double state in [67].

Black hole entropy. Following figure 3, the volume of the timelike-identified region in kinematic space computes the mutual information between the two sides:

$$
I(L, R)=2 S_{\mathrm{BH}}
$$

Referring to the Crofton formula, this equation states that the area of the black hole horizon counts the geodesics that cross the horizon and connect the two sides of the wormhole geometry.

It is instructive to recover this result explicitly in kinematic space by the use of the differential entropy formula. The latter asks for a complete set of geodesics tangent to the horizon, i.e. the lightlike-identified geodesics. Thus, the contour of integration is one full closed lightlike curve in kinematic space, for example $s u_{0}<u \leq u_{0}$ and $v(u)=0$. Substituting this into eq. (2.7), we obtain:

$$
S_{\mathrm{BH}}=-\int_{s u_{0}}^{u_{0}} d u \frac{\partial S(u, 0)}{\partial u}=S\left(s u_{0}, 0\right)-S\left(u_{0}, 0\right)
$$

We recognize this as the difference of the lengths of an $\infty$-wound geodesic and an ' $(\infty-1)$ wound' geodesic. Indeed, taking the boundary-anchored endpoint of the geodesic from $u_{0}$ to $s u_{0}$ winds the already infinitely wound geodesic one additional time. The extra winding 
happens on the horizon of the black hole, which justifies eq. (4.9). Of course, the other closed lightlike-curve in the kinematic space of the BTZ black hole gives a similar result. There, we substitute geodesics $v_{0}<v<s v_{0}$ and $u(v)=0$ into the analogue of $(2.7)$ appropriate for integrating over the $v$ coordinate:

$$
S_{\text {diff }}=\left.\int_{v_{0}}^{s v_{0}} d v \frac{\partial S(u, v)}{\partial v}\right|_{u=u(v)}=S\left(0, s v_{0}\right)-S\left(0, v_{0}\right)=S_{\mathrm{BH}}
$$

Interpreted in MERA, this computation recovers the minimal cut prescription in a novel setting: when the region whose entanglement we compute does not have endpoints. But as a bonus, we have explained why the thermal entropy can be read off from two different minimal cuts, on either side of the timelike-identified region. This is because we have two distinct families of lightlike-identified geodesics, which asymptote to the black hole horizon from either side of the wormhole. Their contributions add up to account for the factor of 2 in eq. (4.8).

Furthermore, our network construction obtains the exact spectrum of the thermofield double state and not just the scaling of the entropy with the number of cuts. Indeed, in ref. [43] our co-authors and we confirmed that the spectrum of the quotient network agrees quantitatively with the entanglement spectrum of the thermofield double state, including the numerical factors in eq. (4.7). This comparison was conducted in the critical Ising model, a decidedly non-holographic theory, in which case the kinematic space ought to be understood as the space of CFT intervals rather than the space of bulk geodesics.

Lightlike-identified geodesics and entwinement. Eqs. (4.9) and (4.10) involve geodesics, which wrap around the black hole horizon. These geodesics do not compute the entanglement entropy of any spatial interval in the thermofield double state. If we allow the use of their lengths in the differential entropy formula, however, we obtain correct geometric quantities, including some information-theoretically meaningful ones such as the entropy of the BTZ black hole [23]. Emboldened by this, ref. [68] named a conjectured CFT avatar of the length of a non-minimal geodesic 'entwinement.' Working in the conical defect geometry, the authors of [68] studied entwinement and concluded that it is related to entanglement among internal degrees of freedom. But an intrinsic definition of entwinement has remained an open question since then.

The quotient MERA manifests the relevance of entwinement in the CFT in the form of a lightlike-identified ray of tensors. Changing any one of these tensors affects the state on the entire CFT circle uniformly. Therefore, we may think of them as acting in the s-wave sector of the CFT, where no further spatial coarse-graining can be performed. Isometries in the lightlike-identified region separate the degrees of freedom which are internally entangled within the s-wave sector on one side from those which carry entanglement with the thermofield image. In the continuous geometry, entwinement is manifested by geodesics that wrap once or more around the black hole. Such 'long geodesics' are sensitive to the internal organization of the CFT degrees of freedom, but also exhibit some degree of localization on the CFT circle. The discrete nature of MERA collapses the entire family of long geodesics into one line of tensors, which live on a lightlike-identified ray. 
Entwinement is related to the structure of the CFT thermal state in the far infrared. At fixed temperature, it should therefore be more important for smaller circle sizes. By eq. (4.7) the effect of entwinement should wash out when $s \rightarrow \infty$ and gain in importance as $k=\log _{2} s$ becomes of order 1. In ref. [43] our co-authors and we confirmed these expectations. In particular, the lightlike-identified region in the quotient network is approximately isometric, with the approximation improving exponentially in $k$. For these reasons, we view the lightlike-identified regions in MERA as a tangible CFT realization of entwinement: because of their nearly isometric character and because their effect is completely delocalized in the CFT.

Spacelike-identified regions. These prepare correlations between spatial regions on one side, just as they would in the vacuum. As unitary transformations between $\mathcal{H}_{\mathrm{IR}} \otimes$ $\mathcal{H}_{\text {frozen }}$ and $\mathcal{H}_{\mathrm{UV}}$, they select a local basis on each side in which the thermofield double state is presented. Different choices of local bases correspond to different conformal frames, distinguished by different UV cutoffs.

\section{Toward excited states: geometry as compression}

The intimate relation between integral geometry and information theory [5] prompted us to look for an analogous structure on the CFT side of the holographic duality. We found it in the MERA tensor network. The two key properties, which MERA shares with kinematic space are the causal structure and the representation of entanglement entropy as a 'flux' through a causal cut (counting lines in MERA and eq. (3.5) in kinematic space). All our arguments originate from these two starting points.

We would like to extend our conclusions beyond the ground state MERA and its sub-networks - the cases discussed in sections 3 and 4 . We begin with the following observations:

1. In the ground state MERA, counting lines that cross a causal cut computes an entanglement entropy only in the optimized network. This feature is not a built-in property of the network; it is an emergent feature that arises after optimization and should not be expected to hold in excited states.

2. The kinematic metric is not rigid. As seen in eq. (2.11), it depends on the state under consideration. When we consider excited states, the kinematic metric can only be relevant to the optimized network whose structure is adjusted for an efficient description of the state.

In consequence, we must look for a flexible network that can incorporate the entanglement pattern in its structure. We propose a holographically motivated generalization of MERA which (a) shares the causal structure of MERA and kinematic space and (b) maintains the approximate relation between entanglement entropies and counting lines on causal cuts. Property (b) will require the state on a causal cut to be an approximate product state, a condition that is generally achievable only in holographic theories. 
In information theoretic terms, such a tensor network is an iterative compression algorithm: it maps the density matrix of every interval to a compressed state on its exclusive causal cone. Conditional mutual information - which for the ground state MERA was the number of isometries in a causal diamond - now provides a local density of compression, namely the net reduction of the local Hilbert space dimension upon an isometric coarse-graining. Details of the construction, properties and limitations of the compression algorithm, as well as new insights about holographic geometries that follow from it, will be presented in an upcoming paper [30].

\section{Discussion}

In this paper, we have argued that the MERA tensor network enjoys a closer relationship to the kinematic space - in a holographic context, the space of bulk geodesics - than it does to the bulk geometry itself. Of course, any such correspondence is at best qualitative in nature; there is no known continuum limit of MERA in which either object can be recovered. Thus, the only criterion for judging a proposed relation between MERA and an emergent geometry is the usefulness of such a proposal - either as inspiration or as a practical tool enabling further progress.

Benefits of the two holographic readings of MERA. In its inspirational aspect, Swingle's original idea to relate MERA directly to the holographic bulk has passed the test of usefulness with flying colors. References $[35,36]$ and the numerous papers that followed them ([31-34, 37-43] and many others) have unveiled a new point of contact between holographic duality and condensed matter theory and offered a novel perspective on the emergence of the bulk spacetime, including its conjectural relation to complexity theory [69].

At the same time, the effort to recast spacetime as a tensor network has consistently led away from the original MERA setup. Attempts to mimic desirable properties of the holographic bulk such as the error correcting property [70] have instead given rise to several novel types of networks [31-34], which differ from MERA in important ways. At present, it is unclear whether these networks can prepare the wavefunction of the CFT ground state. Likewise, the question of how such networks can be extended to encode wavefunctions of excited states and their dual geometries remains open. That said, the AdS/CFT correspondence essentially guarantees the existence of some tensor network, which discretizes the bulk geometry. After all, the change of basis that takes CFT degrees of freedom into low energy effective fields in the bulk can always be presented in the form of a tensor network. That tensor network, however, is not MERA.

If the goal is to discretize the bulk, we should trade MERA for other tools. Conversely, if MERA is the tool, we should find another use for it. This is the philosophy that motivated the present paper; we reasoned that MERA clearly gets something right about conformal field theories, so conformal field theory and the AdS/CFT correspondence stand to gain from insights about MERA if they are properly interpreted. Our contention is that kinematic space supplies the most productive framework for interpreting MERA. 
In the following we list ways in which the relation between MERA and kinematic space has been useful or may become useful in the future:

An improved understanding of MERA. Ref. [43], on which we expanded in section 4, explained how the optimized MERA recovers local conformal invariance. It revealed that building blocks of MERA could be applied locally to perform inhomogeneous coarse-grainings. On a more pedestrian level, it gave an efficient method for converting the ground state MERA to the thermal state MERA. These advances did not rely on the AdS/CFT correspondence, but the holographic duality had brought them to focus.

The kinematic space (understood as the space of CFT intervals) was a useful tool in this work. It gave the initial motivation to consider the quotient in figure 15 and made sense of its decomposition into an isometric and a spectrum-generating part. These findings illustrate the clarifying power of the MERA-kinematic space relation, especially with regard to conformal symmetry.

Fields on kinematic space for a better MERA? The present work focused on one aspect of the integral geometry of the bulk spacetime: recovering lengths from kinematic volumes. Another classic, integral geometric problem - one that has shown up in seismology [8], medical imaging [9] and other physical sciences - is to recover a function on a manifold from its integrals along geodesics [7]. In [11], we studied this problem in the context of the AdS/CFT correspondence. We found that integrals of bulk scalar fields along geodesics are fields in kinematic space which obey the Klein-Gordon equation ${ }^{6}$ (see also [72-75]):

$$
\left(\square_{\text {K.S. }}+m^{2}\right) \int_{\text {geodesic } \gamma} d s \mathcal{O}_{\text {HKLL }}(s) \equiv\left(\square_{\text {K.S. }}+m^{2}\right) \mathcal{B}(\gamma)=0 .
$$

In the CFT, we recognized the kinematic fields $\mathcal{B}(\gamma)$ as OPE blocks-contributions of individual conformal families to operator product expansions of pairs of local operators. This finding is a new entry in the holographic dictionary. It generalizes the Ryu-Takayanagi proposal and sheds a new light on the construction of local bulk operators in AdS/CFT. Indeed, the so-called HKLL (local bulk) operators in AdS [76-79] are related to OPE blocks by an invertible integral transform. This conclusion has some interesting but notyet-understood interplay with the error correcting property of bulk fields emphasized in [70].

The web of connections between conformal field theory, integral geometry and bulk reconstruction must have a counterpart in the language of MERA. In particular, OPE blocks are a particularly convenient operator basis for a CFT in that they automatically include entire conformal multiplets (primary plus descendants) with correct relative weights. OPE blocks also stand out as the obvious operator basis when we put a conformal theory on a lattice. In a cutoff theory every field should be understood to be smeared over a cutoff-sized interval and OPE blocks define the unique conformally invariant way of smearing primaries over intervals.

\footnotetext{
${ }^{6}$ The kinematic Klein-Gordon equation for a metric perturbation is the linearized Einstein equation [71]. In this way, eq. (6.1) encapsulates the derivation of linearized Einstein's equations from the first law of entanglement given in [16].
} 
We believe that OPE blocks - fields on the kinematic space which MERA discretizes - can be used to further improve the Multi-scale Entanglement Renormalization Ansatz. In the present version of MERA, the conformal dimensions and OPE coefficients of primary and descendant operators have to be found independently by numerical optimization. This means that a vast amount of data retrieved from the optimized MERA is known ahead of time from conformal symmetry. These data serve, in effect, as 'check digits' of the optimization problem; see e.g. table I in [50].

If we manage to formulate a MERA-like network in terms of OPE blocks, the optimization problem will be narrowed to finding two pieces of data: the conformal dimensions of the primaries and their OPE coefficients. This would be an enormous simplification, both on a computational and a conceptual level. It would be interesting to examine from this perspective the recent exposition of MERA as a wavelet transform [80]. In the AdS/CFT correspondence, the understanding of HKLL operators as linear combinations of OPE blocks is a promising strategy to convert the MERA network into a network that discretizes the bulk spacetime - so as to upgrade the toy models of [31-34] into realistic calculational tools for CFTs.

Higher dimensional AdS and kinematic spaces. This paper is concerned with understanding MERA in 1+1-dimensional theories. But MERA has also been used for modeling higher dimensional conformal field theories; if it truly carries lessons for the AdS/CFT correspondence, those lessons ought to extend to higher dimensions. Here again an inspiration for progress comes from integral geometry and the OPE blocks. The aforementioned facts about OPE blocks - that they are dual to integrals of bulk fields over homogeneous surfaces, that they obey a version of eq. (6.1) in kinematic space, and that local bulk operators can be simply recovered from them - all continue to hold in higher dimensions. If the 1+1-dimensional MERA can be made to speak the language of OPE blocks, its higher-dimensional cousins will be likely to follow.

It turns out that 1+1-dimensional CFTs have in stock an hors-d'œuvre, which can whet the appetite for higher-dimensional challenges. To understand this point, we have to detach the concept of kinematic space from a preferred time slice of the CFT. Instead, consider kinematic space v2.0, which consists of pairs of CFT points $(x, y)$. For a $d$ dimensional CFT, this is a $2 d$-dimensional space. In $1+1$ CFT dimensions, elements of this generalized kinematic space are still intervals, but they no longer have to live on a time slice of the CFT. The metric of the (now four-dimensional) kinematic space of a $\mathrm{CFT}_{2}$ can be written [11] as

$$
d s^{2}=\frac{\partial^{2} S(x, y)}{\partial x^{\mu} \partial y^{\nu}} d x^{\mu} d y^{\nu},
$$

where $x^{\mu}$ and $y^{\nu}$ are coordinates of the points $x$ and $y$ in the CFT. The kinematic space (2.11) that was the object of our analysis thus far is a slice of (6.2), obtained by setting $x^{0}=y^{0}=$ const.

While our interest in the ground state MERA dictated a focus on that special twodimensional slice of the four-dimensional kinematic space, other slices of (6.2) are also interesting. Consider two space-like 'trajectories' $x^{\mu}(u)$ and $y^{\nu}(v)$ and the induced metric 
on the resulting slice of kinematic space:

$$
d s_{\text {slice }}^{2}=\frac{\partial^{2} S(x, y)}{\partial x^{\mu} \partial y^{\nu}} \frac{d x^{\mu}}{d u} \frac{d y^{\nu}}{d v} d u d v
$$

If $x^{\mu}(u)$ and $y^{\nu}(v)$ follow the same trajectory, metric (6.3) is an analogue of (2.11). Because the trajectory $x^{\mu}(u)$ can be conformally mapped to an equal time slice of the CFT, (6.3) is canonically associated to a local descendant of the CFT vacuum. Following [43], the local descendant can be prepared by the optimized vacuum MERA with certain tensors added or removed. That descendant MERA is a discretization of metric (6.3). But we may also choose $x^{\mu}(u)$ and $y^{\nu}(v)$ to be different from one another. The slice of kinematic space that follows from such a choice can also capture the information about a state, albeit in a way that is delocalized in time! It would be interesting to assemble from the various slices (6.3) a master tensor network, which would discretize the full kinematic space (6.2).

What is not special about holographic CFTs? None of our arguments depends on the CFT having a holographic dual; a holographic CFT with large central charge $c$ fits our story just as well as do $c$ copies of the critical Ising model. To wit, the kinematic space (2.11) and its off-time-slice generalization (6.2) are defined for any conformal field theory and only attain a bonus interpretation as spaces of bulk geodesics when the CFT has a dual geometric description. This may be troubling to some readers who focus primarily on the question: what is special about holographic CFTs?

We believe that it is equally important to ask the complementary question: what is not special about holographic CFTs? Any researcher who has studied or drawn inspiration from the original papers on MERA and the holographic duality $[35,36]$ has implicitly asked the same question. After all, MERA does not use any special properties of holographic theories, it applies equally well to non-holographic CFTs, and has only been used in practical calculations for theories with $\mathcal{O}(1)$ central charge. Consequently, any lessons drawn from MERA can only be a result of conformal symmetry.

This is not a drawback of the program. In studying a complex duality such as the AdS/CFT correspondence, it is crucial to clarify the origins of its various features. Kinematic space and MERA have already been very useful in these respects. Many statements that carry a gravitational flair have by now turned out to be consequences of conformal symmetry. Perhaps the most spectacular example are Einstein's equations linearized about pure AdS [16], which turn out to be kinematic equations of motion (such as 6.1) for the stress tensor OPE block [71]. In highlighting the relation between MERA and kinematic space, we hope to clarify the roles of tensor networks and conformal symmetry in the holographic emergence of AdS spacetimes.

\section{Acknowledgments}

We thank Glen Evenbly, Patrick Hayden, Esperanza Lopez, Don Marolf, Rob Myers, John Preskill, Xiao-Liang Qi, Joan Simón, Leonard Susskind, Brian Swingle and Guifré Vidal for useful discussions. BC, SM and JS thank Caltech, BC thanks the University of Amsterdam 
and the University of Edinburgh, and JS thanks Princeton University for hospitality. BC thanks the organizers of "Holographic duality for condensed matter physics" and KITPCCAS in Beijing. We all thank the organizers of "Quantum Gravity Foundations: UV to IR," "Closing the Entanglement Gap: Quantum Information, Quantum Matter and Quantum Fields," and the Follow-On Program held at KITP (supported in part by the National Science Foundation under Grant No. NSF PHY11-25915), and of "Quantum Information Theory in Quantum Gravity II" meeting held at the Perimeter Institute for Theoretical Physics (supported by the Government of Canada through Industry Canada and by the Province of Ontario through the Ministry of Research and Innovation). BC and JS thank the organizers of the workshop "AdS/CFT and Quantum Gravity" at Centre de Recherches Mathématiques at the University of Montreal. SM was supported in part by an award from the Department of Energy (DOE) Office of Science Graduate Fellowship Program.

Open Access. This article is distributed under the terms of the Creative Commons Attribution License (CC-BY 4.0), which permits any use, distribution and reproduction in any medium, provided the original author(s) and source are credited.

\section{References}

[1] A. Einstein, Erklärung der Perihelbewegung des Merkur aus der allgemeinen Relativitätstheorie, König. Preuss. Akad. Wissen. (Berlin) (1915) 831.

[2] J.M. Maldacena, The large- $N$ limit of superconformal field theories and supergravity, Int. J. Theor. Phys. 38 (1999) 1113 [Adv. Theor. Math. Phys. 2 (1998) 231] [hep-th/9711200] [INSPIRE].

[3] S.D. Mathur, The information paradox: a pedagogical introduction, Class. Quant. Grav. 26 (2009) 224001 [arXiv:0909.1038] [INSPIRE].

[4] A. Almheiri, D. Marolf, J. Polchinski and J. Sully, Black holes: complementarity or firewalls?, JHEP 02 (2013) 062 [arXiv:1207.3123] [INSPIRE].

[5] B. Czech, L. Lamprou, S. McCandlish and J. Sully, Integral geometry and holography, JHEP 10 (2015) 175 [arXiv: 1505.05515] [INSPIRE].

[6] L. Santaló, Integral geometry and geometric probability, Cambridge University Press, Cambridge U.K. (1976).

[7] S. Helgason, Radon transform, Birkhäuser, Boston U.S.A. (1983)

[8] G. Beylkin, Discrete Radon transform, IEEE Trans. Acoust. Speech 35 (1987) 162.

[9] The Nobel prize in physiology or medicine 1979, http://www.nobelprize.org Nobel Media AB (2014), retriveved on 26 September (2015).

[10] J. Lin, M. Marcolli, H. Ooguri and B. Stoica, Locality of gravitational systems from entanglement of conformal field theories, Phys. Rev. Lett. 114 (2015) 221601 [arXiv: 1412.1879] [INSPIRE].

[11] B. Czech, L. Lamprou, S. McCandlish, B. Mosk and J. Sully, A stereoscopic look into the bulk, arXiv: 1604.03110 [INSPIRE].

[12] S. Ryu and T. Takayanagi, Holographic derivation of entanglement entropy from AdS/CFT, Phys. Rev. Lett. 96 (2006) 181602 [hep-th/0603001] [INSPIRE]. 
[13] S. Ryu and T. Takayanagi, Aspects of holographic entanglement entropy, JHEP 08 (2006) 045 [hep-th/0605073] [INSPIRE].

[14] V.E. Hubeny, M. Rangamani and T. Takayanagi, A covariant holographic entanglement entropy proposal, JHEP 07 (2007) 062 [arXiv:0705.0016] [INSPIRE].

[15] N. Lashkari, C. Rabideau, P. Sabella-Garnier and M. Van Raamsdonk, Inviolable energy conditions from entanglement inequalities, JHEP 06 (2015) 067 [arXiv:1412.3514] [INSPIRE].

[16] N. Lashkari, M.B. McDermott and M. Van Raamsdonk, Gravitational dynamics from entanglement 'thermodynamics', JHEP 04 (2014) 195 [arXiv: 1308.3716] [INSPIRE].

[17] T. Faulkner, M. Guica, T. Hartman, R.C. Myers and M. Van Raamsdonk, Gravitation from entanglement in holographic CFTs, JHEP 03 (2014) 051 [arXiv:1312.7856] [INSPIRE].

[18] B. Swingle and M. Van Raamsdonk, Universality of gravity from entanglement, arXiv: 1405.2933 [INSPIRE].

[19] N. Lashkari and M. Van Raamsdonk, Canonical energy is quantum fisher information, JHEP 04 (2016) 153 [arXiv: 1508.00897] [INSPIRE].

[20] V. Balasubramanian, B.D. Chowdhury, B. Czech, J. de Boer and M.P. Heller, Bulk curves from boundary data in holography, Phys. Rev. D 89 (2014) 086004 [arXiv:1310.4204] [INSPIRE].

[21] M. Headrick, R.C. Myers and J. Wien, Holographic holes and differential entropy, JHEP 10 (2014) 149 [arXiv: 1408.4770] [INSPIRE].

[22] B. Czech, P. Hayden, N. Lashkari and B. Swingle, The information theoretic interpretation of the length of a curve, JHEP 06 (2015) 157 [arXiv:1410.1540] [INSPIRE].

[23] B. Czech and L. Lamprou, Holographic definition of points and distances, Phys. Rev. D 90 (2014) 106005 [arXiv: 1409.4473] [INSPIRE].

[24] M. Van Raamsdonk, Comments on quantum gravity and entanglement, arXiv:0907.2939 [INSPIRE].

[25] M. Van Raamsdonk, Building up spacetime with quantum entanglement, Gen. Rel. Grav. 42 (2010) 2323 [arXiv: 1005. 3035] [inSPIRE].

[26] G. Leclerc de Bouffon, Editor's note concerning a lecture given 1733 by Mr. Le Clerc de Buffon to the Royal Academy of Sciences in Paris, Histoire del'Acad. Roy. des Sci. (1733) 43.

[27] G. Vidal, Entanglement renormalization, Phys. Rev. Lett. 99 (2007) 220405 [cond-mat/0512165] [INSPIRE].

[28] G. Vidal, A class of quantum many-body states that can be efficiently simulated, Phys. Rev. Lett. 101 (2008) 110501 [cond-mat/0610099].

[29] C. Beny, Causal structure of the entanglement renormalization ansatz, New J. Phys. 15 (2013) 023020 [arXiv: 1110.4872] [INSPIRE].

[30] B. Czech, P. Hayden, L. Lamprou, S. McCandlish and J. Sully, Geometry as a compression algorithm, to appear.

[31] F. Pastawski, B. Yoshida, D. Harlow and J. Preskill, Holographic quantum error-correcting codes: toy models for the bulk/boundary correspondence, JHEP 06 (2015) 149 [arXiv: 1503.06237] [INSPIRE]. 
[32] Z. Yang, P. Hayden and X.-L. Qi, Bidirectional holographic codes and sub-AdS locality, JHEP 01 (2016) 175 [arXiv: 1510.03784] [INSPIRE].

[33] P. Hayden, S. Nezami, X.-L. Qi, N. Thomas, M. Walter and Z. Yang, Holographic duality from random tensor networks, arXiv:1601.01694 [INSPIRE].

[34] A. Bhattacharyya, Z.-S. Gao, L.-Y. Hung and S.-N. Liu, Exploring the tensor networks/AdS correspondence, arXiv:1606.00621 [INSPIRE].

[35] B. Swingle, Entanglement renormalization and holography, Phys. Rev. D 86 (2012) 065007 [arXiv:0905.1317] [INSPIRE].

[36] B. Swingle, Constructing holographic spacetimes using entanglement renormalization, arXiv:1209.3304 [INSPIRE].

[37] M. Nozaki, S. Ryu and T. Takayanagi, Holographic geometry of entanglement renormalization in quantum field theories, JHEP 10 (2012) 193 [arXiv:1208.3469] [INSPIRE].

[38] T. Hartman and J. Maldacena, Time evolution of entanglement entropy from black hole interiors, JHEP 05 (2013) 014 [arXiv: 1303.1080] [INSPIRE].

[39] X.-L. Qi, Exact holographic mapping and emergent space-time geometry, arXiv:1309.6282 [INSPIRE].

[40] D.A. Roberts, D. Stanford and L. Susskind, Localized shocks, JHEP 03 (2015) 051 [arXiv: 1409.8180] [INSPIRE].

[41] M. Miyaji, T. Numasawa, N. Shiba, T. Takayanagi and K. Watanabe, Continuous Multiscale Entanglement Renormalization Ansatz as Holographic Surface-State Correspondence, Phys. Rev. Lett. 115 (2015) 171602 [arXiv:1506.01353] [INSPIRE].

[42] D. Marolf, H. Maxfield, A. Peach and S.F. Ross, Hot multiboundary wormholes from bipartite entanglement, Class. Quant. Grav. 32 (2015) 215006 [arXiv: 1506.04128] [INSPIRE].

[43] B. Czech et al., A tensor network quotient takes the vacuum to the thermal state, arXiv: 1510.07637 [INSPIRE].

[44] G. Solanes, Integral geometry and curvature integrals in hyperbolic space, Ph.D. thesis, Universitat Autonoma de Barcelona, Barcelona, Spain (2003).

[45] E.H. Lieb and M.B. Ruskai, Proof of the strong subadditivity of quantum-mechanical entropy, J. Math. Phys. 14 (1973) 1938 [InSPIRE].

[46] G. Vidal, Entanglement renormalization: an introduction, arXiv:0912.1651.

[47] G. Evenbly and G. Vidal, Quantum criticality with the multi-scale entanglement renormalization ansatz, arXiv:1109.5334.

[48] G. Evenbly and G. Vidal, Algorithms for entanglement renormalization, Phys. Rev. B 79 (2009) 144108 [arXiv:0707.1454].

[49] R.N.C. Pfeifer, G. Evenbly and G. Vidal, Entanglement renormalization, scale invariance and quantum criticality, Phys. Rev. A 79 (2009) 040301 [arXiv:0810.0580] [INSPIRE].

[50] G. Evenbly, P. Corboz and G. Vidal, Non-local scaling operators with entanglement renormalization, Phys. Rev. B 82 (2010) 132411 [arXiv:0912.2166].

[51] P. Calabrese and J.L. Cardy, Entanglement entropy and quantum field theory, J. Stat. Mech. 0406 (2004) P06002 [hep-th/0405152] [INSPIRE]. 
[52] D.N. Page, Average entropy of a subsystem, Phys. Rev. Lett. 71 (1993) 1291 [gr-qc/9305007] [INSPIRE].

[53] G. Evenbly, private communication.

[54] G. Evenbly and G. Vidal, A theory of minimal updates in holography, Phys. Rev. B 91 (2015) 205119 [arXiv: 1307.0831] [InSPIRE].

[55] N. Bao et al., Consistency conditions for an AdS multiscale entanglement renormalization ansatz correspondence, Phys. Rev. D 91 (2015) 125036 [arXiv:1504.06632] [INSPIRE].

[56] R.C. Myers, J. Rao and S. Sugishita, Holographic holes in higher dimensions, JHEP 06 (2014) 044 [arXiv:1403.3416] [INSPIRE].

[57] M. Miyaji and T. Takayanagi, Surface/state correspondence as a generalized holography, PTEP 2015 (2015) 073B03 [arXiv: 1503.03542] [INSPIRE].

[58] E.T. Akhmedov, A remark on the AdS/CFT correspondence and the renormalization group flow, Phys. Lett. B 442 (1998) 152 [hep-th/9806217] [INSPIRE].

[59] V. Balasubramanian and P. Kraus, Space-time and the holographic renormalization group, Phys. Rev. Lett. 83 (1999) 3605 [hep-th/9903190] [INSPIRE].

[60] J. de Boer, E.P. Verlinde and H.L. Verlinde, On the holographic renormalization group, JHEP 08 (2000) 003 [hep-th/9912012] [INSPIRE].

[61] M. Bañados, Three-dimensional quantum geometry and black holes, hep-th/9901148 [INSPIRE].

[62] G. Mandal, R. Sinha and N. Sorokhaibam, The inside outs of $A d S_{3} / \mathrm{CFT}_{2}$ : exact AdS wormholes with entangled CFT duals, JHEP 01 (2015) 036 [arXiv:1405.6695] [INSPIRE].

[63] M.M. Sheikh-Jabbari and H. Yavartanoo, Excitation entanglement entropy in $2 D$ conformal field theories, arXiv:1605.00341 [INSPIRE].

[64] M. Bañados, M. Henneaux, C. Teitelboim and J. Zanelli, Geometry of the $(2+1)$ black hole, Phys. Rev. D 48 (1993) 1506 [Erratum ibid. D 88 (2013) 069902] [gr-qc/9302012] [INSPIRE].

[65] V. Balasubramanian, P. Hayden, A. Maloney, D. Marolf and S.F. Ross, Multiboundary wormholes and holographic entanglement, Class. Quant. Grav. 31 (2014) 185015 [arXiv: 1406.2663] [INSPIRE].

[66] A. de la Fuente and R. Sundrum, Holography of the BTZ black hole, inside and out, JHEP 09 (2014) 073 [arXiv: 1307.7738] [INSPIRE].

[67] P. Kraus, H. Ooguri and S. Shenker, Inside the horizon with AdS/CFT, Phys. Rev. D 67 (2003) 124022 [hep-th/0212277] [INSPIRE].

[68] V. Balasubramanian, B.D. Chowdhury, B. Czech and J. de Boer, Entwinement and the emergence of spacetime, JHEP 01 (2015) 048 [arXiv: 1406.5859] [INSPIRE].

[69] A.R. Brown, D.A. Roberts, L. Susskind, B. Swingle and Y. Zhao, Holographic complexity equals bulk action?, Phys. Rev. Lett. 116 (2016) 191301 [arXiv:1509.07876] [INSPIRE].

[70] A. Almheiri, X. Dong and D. Harlow, Bulk locality and quantum error correction in AdS/CFT, JHEP 04 (2015) 163 [arXiv:1411.7041] [INSPIRE].

[71] B. Czech, L. Lamprou, S. McCandlish, B. Mosk and J. Sully, Einstein equations from kinematic space, to appear. 
[72] M. Nozaki, T. Numasawa, A. Prudenziati and T. Takayanagi, Dynamics of entanglement entropy from Einstein equation, Phys. Rev. D 88 (2013) 026012 [arXiv:1304.7100] [INSPIRE].

[73] J. Bhattacharya and T. Takayanagi, Entropic counterpart of perturbative Einstein equation, JHEP 10 (2013) 219 [arXiv:1308.3792] [INSPIRE].

[74] J. de Boer, M.P. Heller, R.C. Myers and Y. Neiman, Holographic de Sitter geometry from entanglement in conformal field theory, Phys. Rev. Lett. 116 (2016) 061602 [arXiv: 1509.00113] [INSPIRE].

[75] J. de Boer, F.M. Haehl, M.P. Heller and R.C. Myers, Entanglement, holography and causal diamonds, arXiv: 1606.03307 [INSPIRE].

[76] T. Banks, M.R. Douglas, G.T. Horowitz and E.J. Martinec, AdS dynamics from conformal field theory, hep-th/9808016 [INSPIRE].

[77] V. Balasubramanian, P. Kraus, A.E. Lawrence and S.P. Trivedi, Holographic probes of anti-de Sitter space-times, Phys. Rev. D 59 (1999) 104021 [hep-th/9808017] [INSPIRE].

[78] I. Bena, On the construction of local fields in the bulk of $A d S_{5}$ and other spaces, Phys. Rev. D 62 (2000) 066007 [hep-th/9905186] [INSPIRE].

[79] A. Hamilton, D.N. Kabat, G. Lifschytz and D.A. Lowe, Local bulk operators in AdS/CFT: a boundary view of horizons and locality, Phys. Rev. D 73 (2006) 086003 [hep-th/0506118] [INSPIRE].

[80] G. Evenbly and S.R. White, Entanglement renormalization and wavelets, Phys. Rev. Lett. 116 (2016) 140403 [arXiv: 1602.01166] [INSPIRE]. 